\title{
Discrete Conformal Equivalence of Polyhedral Surfaces
}

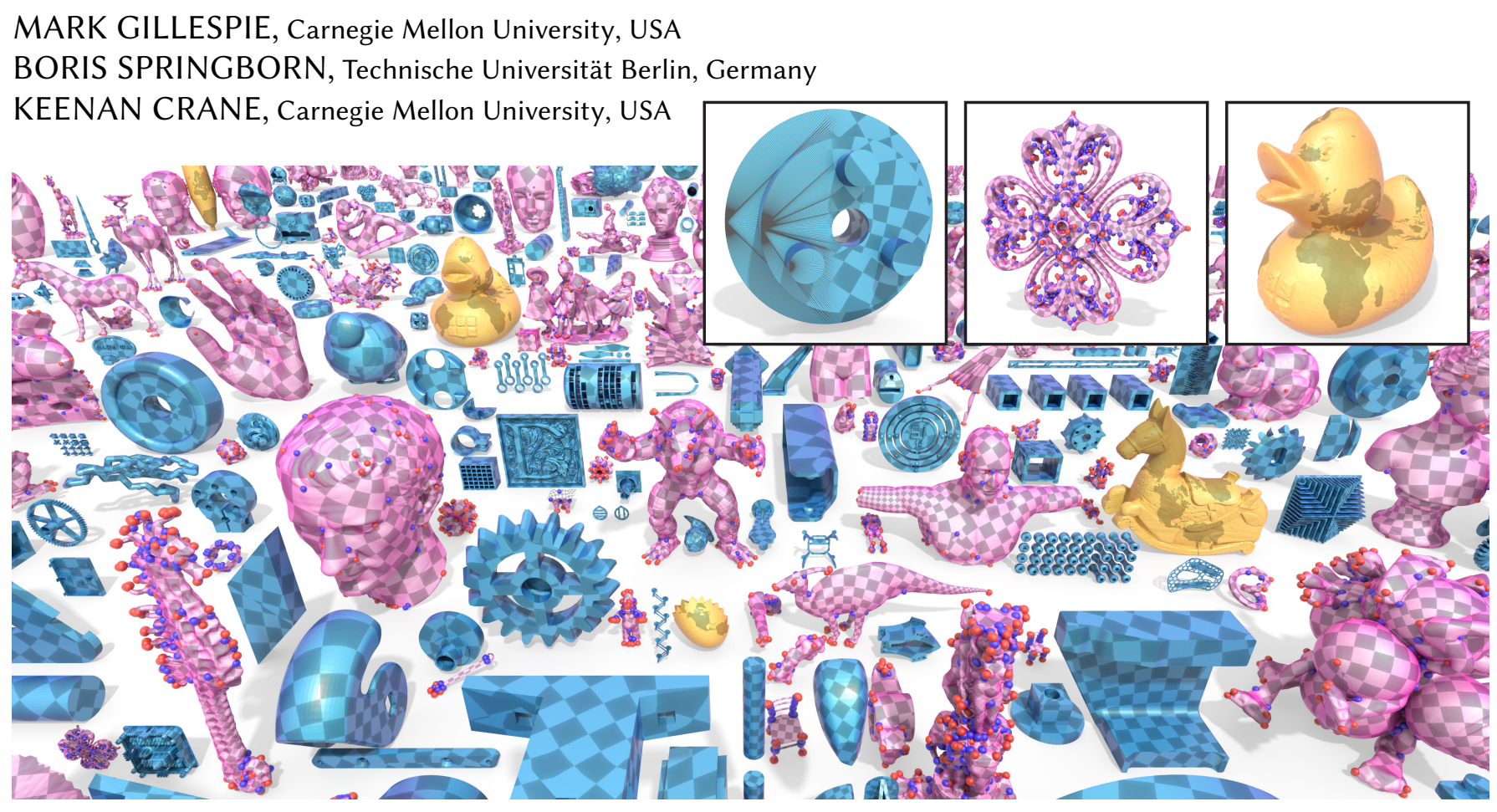

Fig. 1. Our method computes locally injective, discretely conformal maps even for near-degenerate triangulations (turquoise meshes) and extremely difficult configurations of cone singularities (magenta meshes). We also compute globally bijective conformal maps to the sphere (yellow meshes).

\begin{abstract}
This paper describes a numerical method for surface parameterization, yielding maps that are locally injective and discretely conformal in an exact sense. Unlike previous methods for discrete conformal parameterization, the method is guaranteed to work for any manifold triangle mesh, with no restrictions on triangulatiothat each task can be formulated as a convex problem where the triangulation is allowed to change-we complete the picture by introducing the machinery needed to actually construct a discrete conformal map. In particular, we introduce a new scheme for tracking correspondence between triangulations based on normal coordinates, and a new interpolation procedure based on layout in the light cone. Stress tests involving difficult cone configurations and near-degenerate triangulations indicate that the method is extremely robust in practice, and provides high-quality interpolation even on meshes with poor elements.
\end{abstract}

CCS Concepts: • Mathematics of computing $\rightarrow$ Mesh generation.

Additional Key Words and Phrases: conformal parameterization, injective mapping, discrete differential geometry

Authors' addresses: Mark Gillespie, Carnegie Mellon University, USA; Boris Springborn, Technische Universität Berlin, Germany; Keenan Crane, Carnegie Mellon University, USA.

\section{(c) ()}

This work is licensed under a Creative Commons Attribution International 4.0 License.

(c) 2021 Copyright held by the owner/author(s).

0730-0301/2021/8-ART103

https://doi.org/10.1145/3450626.3459763

\section{ACM Reference Format:}

Mark Gillespie, Boris Springborn, and Keenan Crane. 2021. Discrete Conformal Equivalence of Polyhedral Surfaces. ACM Trans. Graph. 40, 4, Article 103 (August 2021), 20 pages. https://doi.org/10.1145/3450626.3459763

\section{INTRODUCTION}

Angle-preserving or conformal maps play a key role in applied geometry [Gu et al. 2020], ranging from biological shape analysis to digital fabrication to machine learning [Koehl and Hass 2015; Konaković et al. 2016; Maron et al. 2017]. Conformal maps are desirable for many reasons: they provide canonical mappings between shapes [Lipman and Daubechies 2011; Baden et al. 2018]; typically involve only sparse linear systems or easy convex problems (hence scale to very large meshes); and provide both low angle and area distortion when enriched with well-placed cuts or cone singularities [Kharevych et al. 2006; Ben-Chen et al. 2008; Sharp and Crane 2018; Soliman et al. 2018].

To date, however, conformal mapping algorithms fail to guarantee that a valid map is always found: if the input surface is too poorly triangulated, or the target curvatures are too extreme, existing methods find a map that is not locally injective, or simply fail to find any map at all. Such failures hinder the reliability of broader geometry processing algorithms that depend on conformal maps. 


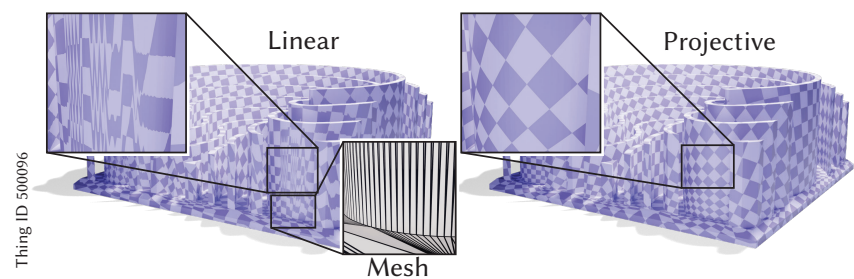

Fig. 3. For meshes with low-quality elements, standard linear interpolation yields a poor conformal map (left). We describe how to perform projective interpolation across triangulations, yielding a much nicer map (right).

In the smooth setting, existence of conformal maps is guaranteed by the uniformization theorem [Abikoff 1981]. Very recently, $\mathrm{Gu}$ et al. [2018a,b] and Springborn [2019] established an analogous discrete uniformization theorem for triangle meshes. However, these theoretical results fall short of providing practical algorithms, since they do not describe how to construct the mapping between the input and target domain. We develop the first end-to-end algorithm for computing and evaluating this map-in particular, we provide:

- a novel combinatorial data structure for tracking correspondence between different triangulations (Section 5),

- a new interpolation scheme for evaluating the discrete conformal map based on the light cone (Section 6), and

- critical details needed to implement discrete uniformization including a careful treatment of numerics, boundary conditions, and subtleties of the spherical case.

Our optimization procedure is a simple modification of the CETM algorithm (from Springborn et al. [2008], Conformal Equivalence of Triangle Meshes): we minimize the same energy, but evaluate it on a triangulation that changes according to the current scale factors. However, since the triangulation may now change, this procedure does not yield an explicit parameterization of the input. To improve the quality of the map, we also flip the input to an intrinsic Delaunay triangulation. The main difficulty in developing a practical algorithm is therefore tracking and evaluating the correspondence between three triangulations-Figure 2 gives an overview of the whole process.

\section{RELATED WORK}

\subsection{Discrete Conformal Equivalence}

In the smooth setting, conformal maps preserve angles-naïvely, one might therefore require that for triangle meshes, discrete conformal maps preserve the angles at all cor-

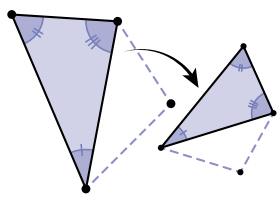
ners. However, this condition is far too rigid: since each triangle can only scale and rotate, its neighbors-and in turn, the entire surface-may only scale by a constant amount. As a result, many other notions of discrete conformal maps have been explored; Crane [2020] gives a detailed account.

A particularly successful approach is the notion of discrete conformal equivalence. In the smooth setting, two Riemannian metrics $g, \tilde{g}$ (which determine angles) are conformally equivalent if they are related by a positive scaling $\tilde{g}=e^{2 u} g$ for some real-valued function $u$. On a triangle mesh, the Riemannian metric is captured by the lengths $\ell_{i j}$ of all edges $i j$, and two sets of lengths $\ell, \tilde{\ell}$ are called discretely conformally equivalent if

$$
\tilde{\ell}_{i j}=e^{\left(u_{i}+u_{j}\right) / 2} \ell_{i j}
$$

for some assignment of scale factors $u_{i} \in \mathbb{R}$

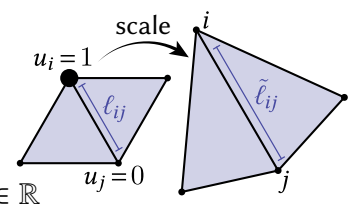
to vertices $i$ [Roček and Williams 1984; Luo 2004]. This innocentlooking definition leads to a rich discrete theory which is just as flexible as the smooth one [Bobenko et al. 2015]. Bücking [2016, 2018 ] and $\mathrm{Gu}$ et al. [2019] consider convergence under refinement.

\subsubsection{Discrete Uniformization.}

Conformal equivalence offers an appealing strategy for parameterization: rather than solve directly for a map to the plane, first find scale factors that describe a discretely conformally equivalent flat surface-perhaps with target angle defects $\Omega_{i}^{*}$ prescribed at just a few isolated cone points (Figure 4, top right). This new surface is then cut open and unfolded into the plane (Figure 4, bottom). In the smooth setting, existence of such scale factors is guaranteed by the uniformization theorem [Abikoff 1981]

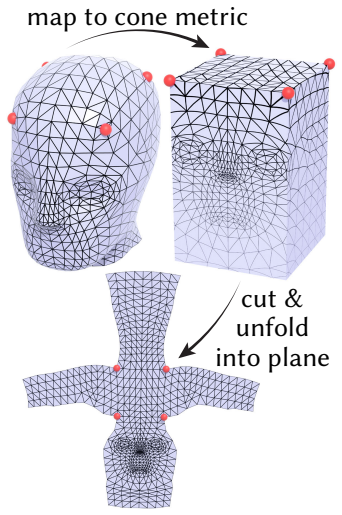

Fig. 4. Conformal parameteriand its generalization to cone metrics [Troyanov 1991]. In the discrete setting, however, there is a critical problem: for a fixed triangulation, there may be no scale factors that achieve the target

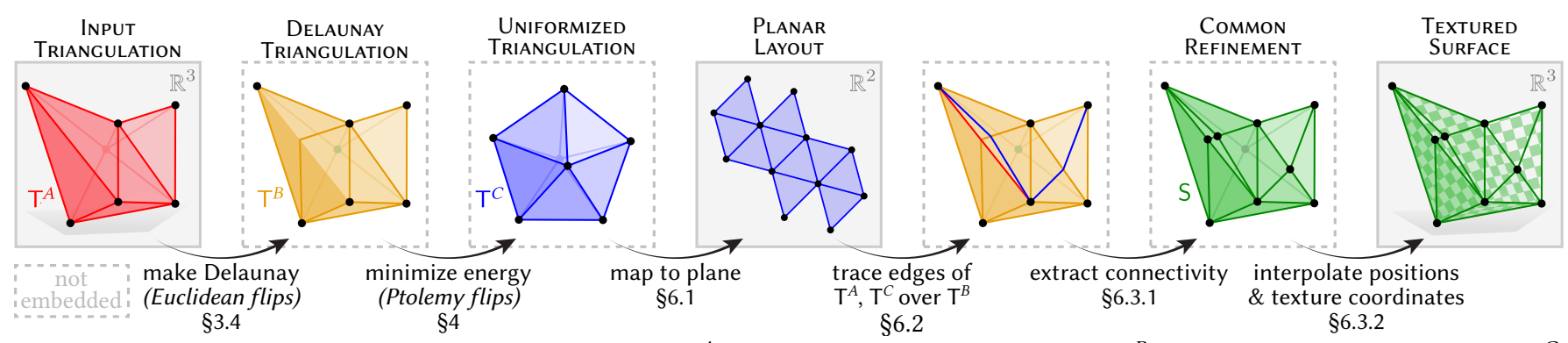

Fig. 2. Steps of our algorithm. Throughout we color the input mesh $\mathrm{T}^{A}$ red, its intrinsic Delaunay triangulation $\mathrm{T}^{B}$ yellow, the uniformized triangulation $\mathrm{T}^{C}$ blue, and the common refinement $S$ of all three green. (Note: triangulations in dashed boxes are purely intrinsic and never actually embedded in $\mathbb{R}^{n}$.) 

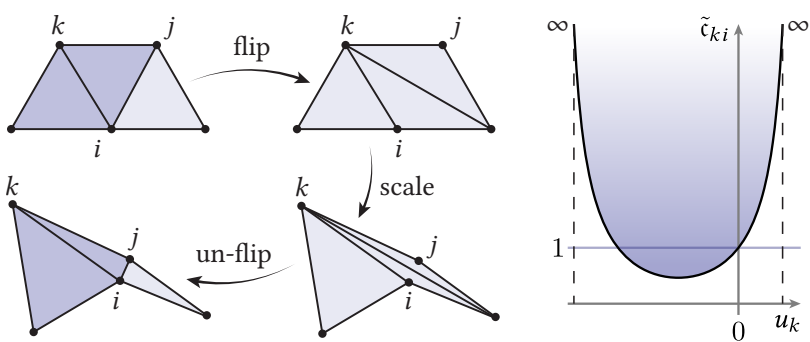

Fig. 5. Performing Euclidean edge flips at arbitrary moments in the flow can badly distort the conformal structure. Here, we flip edge $i j$, scale edges incident on $k$ by a factor $e^{u_{k} / 2}$, and undo the flip. The cross ratio $\tilde{c}_{k i}$ of edge $k i$ (Equation 4) is not preserved, and in fact can take almost any value.

angle defects. One must therefore adopt an expanded notion of discrete conformal equivalence that allows the triangulation to change (Section 2.1.2).

To actually compute the scale factors, Luo [2004] proposed the discrete Yamabe flow

$$
\frac{d}{d t} u_{i}(t)=\Omega_{i}^{*}-\widetilde{\Omega}_{i}(t) .
$$

Here $\widetilde{\Omega}_{i}(t)$ are the angle defects induced by the scale factors $u(t)$ However, since there may be no scale factors that achieve the target angle defects, this flow can fail to reach a critical point $\frac{d}{d t} u_{i}=0$, where $\widetilde{\Omega}_{i}=\Omega_{i}^{*}$. In this case, the scaled edge lengths $\tilde{\ell}$ will eventually violate the triangle inequality-at which point the flow becomes illdefined and cannot continue. Springborn et al. [2008] and Bobenko et al. [2015] describe this flow as gradient descent on an explicit convex energy $\mathcal{E}$, leading to the more efficient, 2nd-order CETM algorithm. CETM extends $\mathcal{E}$ to be well-defined even for invalid edge lengths-but if the minimizer is found in this extended region, it fails to describe a valid parameterization (Figure 25).

Flipping Edges. Luo [2004] conjectured that degenerate triangles might be avoided by applying Euclidean edge flips at the exact moment when triangles degenerate, as implemented by Campen and Zorin [2017b, Section 7.3.1], but this idea has two fatal flaws. First, mixing flips with vertex scaling can yield lengths that are not conformally equivalent to the original ones (Figure 5). Second, it can cause discontinuities in the value of $\mathcal{E}$, voiding any guarantee that the flow will converge (Figure 7). This lack of guarantees is a problem even for methods that care only about injectivity, and not conformal maps [Chien et al. 2016; Campen and Zorin 2017b,a; Campen et al. 2019]. Likewise, the generalized method of Chen et al. [2016, Algorithm 1] takes a step of arbitrary size before performing power Delaunay flips, and [Yu et al. 2017, Algorithm 1] takes an arbitrary step before performing Euclidean flips. Both algorithms can hence distort conformal structure, or worse, produce edge lengths that violate the triangle inequality-at which point the flow is undefined and cannot continue. Our use of Ptolemy flips ensures the flow is always well-defined and exactly preserves the conformal structure (see Section 3.3.4, and the use of Algorithm 11 within Algorithm 4)

2.1.2 Variable Triangulations. A recent theoretical breakthrough is a notion of discrete conformal equivalence that does not depend on how a polyhedral surface is triangulated (Figure 6), along with

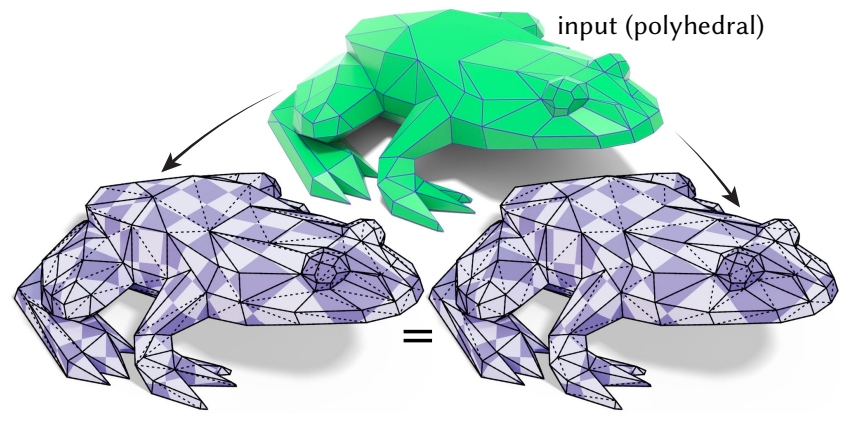

Fig. 6. We adopt a notion of conformal equivalence that yields the same discrete conformal map, no matter how the input polyhedral surface is triangulated. Here a mesh with planar faces is triangulated two different ways, yielding identical results.

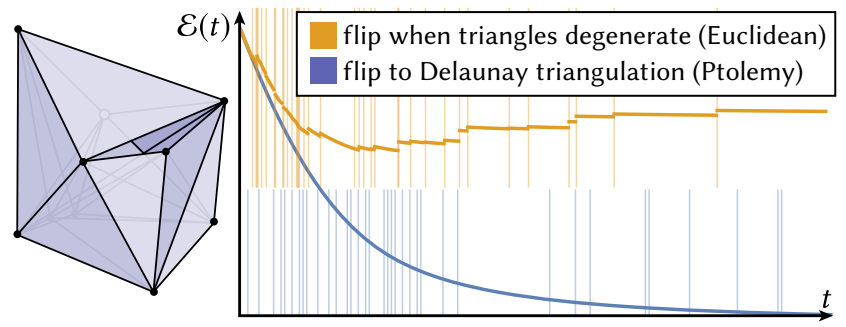

Fig. 7. Flipping edges when triangles degenerate causes the energy $\mathcal{E}$ to jump discontinuously-voiding any guarantee of convergence (top). In contrast, flipping to Delaunay via Ptolemy flips before evaluating the energy ensures that we always reach the correct solution (bottom). Here we consider a coarse double torus with target angle defects $+3 \pi / 4$ at all but one vertex, which has large negative curvature. We take small steps to clearly plot the energy; vertical lines indicate flip times.

associated discrete uniformization theorems for the Euclidean [Gu et al. 2018a], hyperbolic [Gu et al. 2018b], and spherical [Springborn 2019] cases. This work is intimately linked to realization results for ideal hyperbolic polyhedra [Rivin 1994; Fillastre 2008; Prosanov 2020]. The theorems guarantee one can always find a conformally equivalent triangulation with prescribed angle defects $\Omega^{*}$, so long as they satisfy Gauss-Bonnet. This solution is unique up to scale (Euclidean case) or Möbius transformations (spherical case).

There are two equivalent definitions of discrete conformal equivalence-a key idea introduced by $\mathrm{Gu}$ et al. [2018a] is to consider an intrinsic Delaunay triangulation of the input (Section 3.4).

One definition is that two Delaunay triangulations are conformally equivalent if they are related by an alternating sequence of vertex scalings (Equation 1) and concyclic Euclidean edge flips (Figure 9), which main-

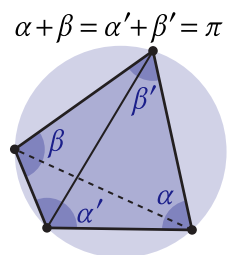

Fig. 9. Either triangulation of a circular quad satisfies the local Delautain the Delaunay property [Gu et al. 2018a, nay property $\alpha+\beta \leq \pi$. Definition 1.1]. Algorithms that adopt this definition must stop and flip whenever two triangles become concylic. Wu [2014] shows that only finitely many flips are needed, ensuring that computation 


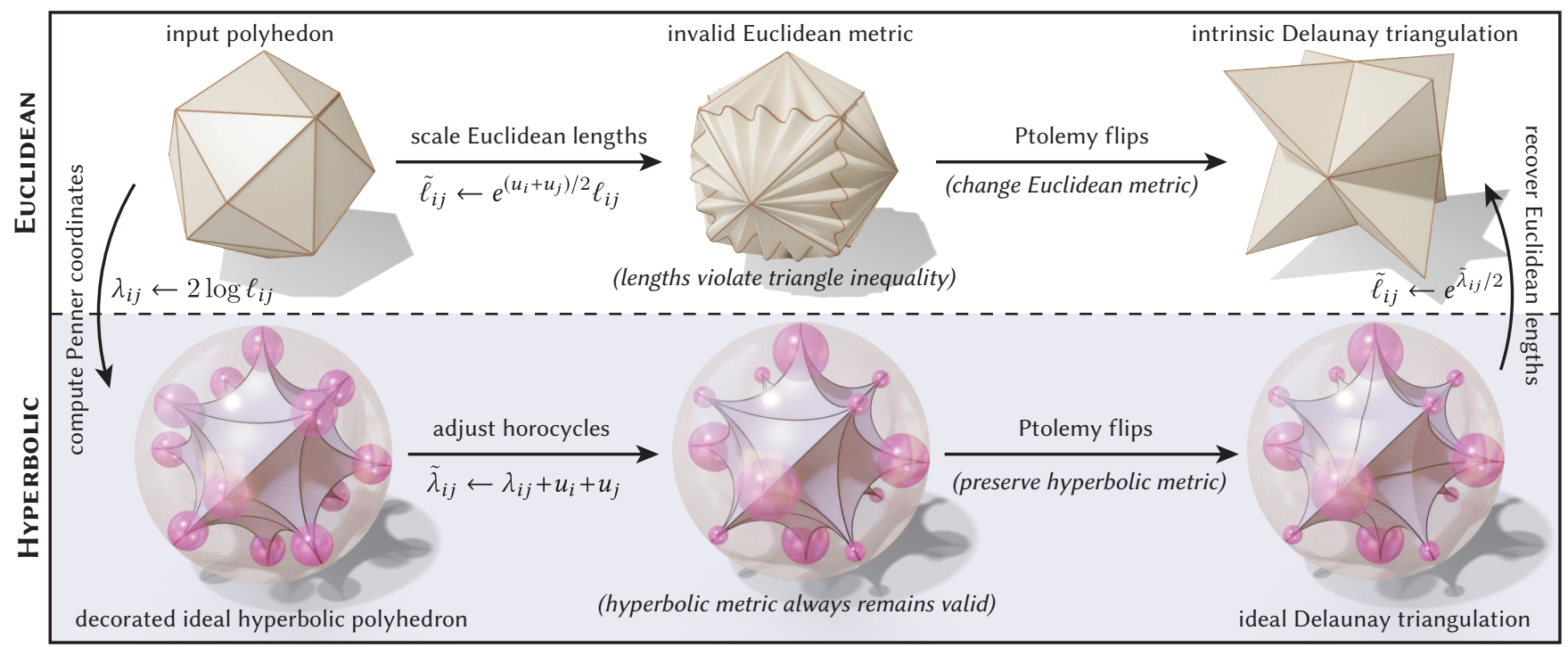

Fig. 8. Top: Triangle meshes with different connectivity (but the same vertices) are considered discretely conformally equivalent if they are the same up to a conformal rescaling of edge lengths, followed by Ptolemy edge flips to a Delaunay triangulation. Bottom: This definition, and the use of Ptolemy (rather than Euclidean) edge flips, arises from a hyperbolic perspective, where we simply retriangulate a hyperbolic polyhedron without changing its geometry.

terminates. Sun et al. [2015] present an implementation of such a scheme, but do not evaluate the pointwise map between the domain and target (as needed for, e.g., texture mapping or remeshing).

We adopt an alternative definition which is theoretically equivalentthough this is far from obvious: the two triangulations are discretely conformally equivalent if they describe the same ideal hyperbolic polyhedron [Bobenko et al. 2015, Definition 5.1.4]. As observed by Springborn [2019], a discretely conformally equivalent triangulation can be obtained by applying an arbitrary vertex scaling, then flipping to a Delanuay triangulation via Ptolemy flips (Section 3.3.4), rather than ordinary Euclidean flips-see Figure 8, top. Since Ptolemy flips are well-defined even when edge lengths fail to satisfy the triangle inequality, one need not worry about maintaining a valid Euclidean metric, nor about triangles being concyclic: at any moment, one can simply scale to an invalid metric, then flip to a valid one. This procedure always works, because it corresponds to retriangulating the associated ideal hyperbolic polyhedron (Figure 8, bottom). Concurrent work by Campen et al. [2021] also takes this approach.

By adopting this definition, we cast discrete conformal parameterization as an unconstrained convex optimization problem where the only variables are the scale factors $u_{i}$. The optimizer need not worry about edge flips, which appear only within a black-box callback routine that evaluates the energy and its derivatives. Moreover, we can use a 2nd-order Newton method to achieve fast convergence, since the energy we minimize is twice continuously differentiable $\left(C^{2}\right)$ even across different triangulations, and the Hessian is easy to compute (just the cotan-Laplacian). Overall this approach is generally faster than stopping to perform flips (see Figure 10 and Section 8.3), and also accommodates the more difficult spherical case, which involves additional bounds constraints (Section 7).

\subsection{Discrete Conformal Mapping}

The triangulation produced by uniformization cannot be used in most applications unless we know how to map data back to the input mesh. Two basic strategies have been developed for this purpose. Fisher et al. [2007] maintain an explicit mesh of the common refinement of two triangulations, guaranteeing correct connectivity (Sun et al. [2015] adopt a similar approach). Sharp et al. [2019b] observe that explicit encodings incur significant cost, and instead implicitly encode correspondence via signposts at vertices. This floating-point encoding can however fail to describe correct connectivity in extreme situations (such as Figure 27). We provide the best of both worlds: an implicit, integer-based encoding that can be updated without resolving intersections, yet guarantees the right connectivity (Section 5). This encoding is based on normal coordinates, a tool

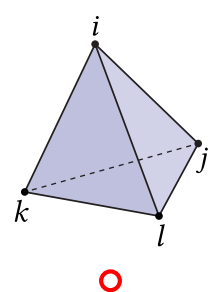

concyclic flips [Sun et al 2015]
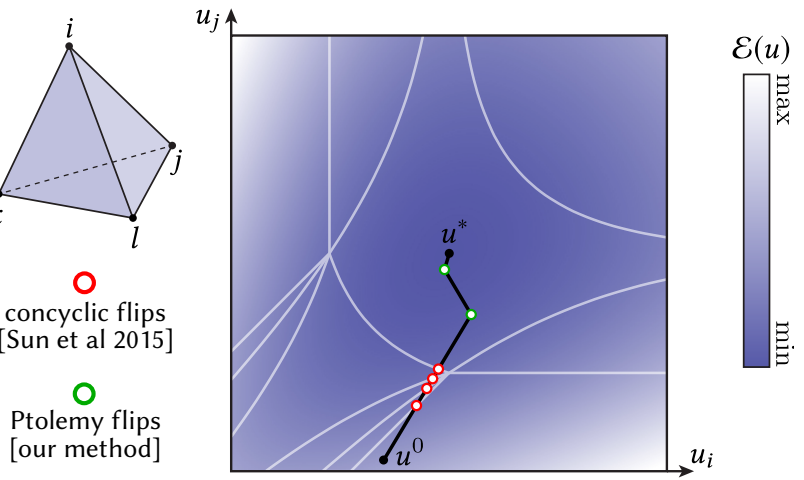

Fig. 10. A slice of the energy landscape for a tetrahedron. Each conformal scaling $u$ induces a Delaunay triangulation-white curves delineate regions with a common triangulation. Previous algorithms must stop and flip at each region boundary (where triangles become concyclic), whereas we can flip at any moment-since Ptolemy flips commute with scaling. 
from 3-manifold geometry [Kneser 1929; Haken 1961] and computational topology [Schaefer et al. 2002; Agol et al. 2006; Erickson and Nayyeri 2013]. We enrich this construction with a combinatorial analogue of signposts, which we call roundabouts (Section 5).

The other question is how to interpolate data across triangulations, such as vertex or texture coordinates. The natural choice for discrete conformal maps is to use piecewise projective interpolation [Bobenko et al. 2015], which can be implemented via standard homogeneous coordinates [Springborn et al. 2008, Section 3.4]. We extend this idea to variable triangulations by laying out triangles in the light cone rather than the Euclidean plane (see Section 6.0.1).

Importantly, our approach to discrete conformal mapping depends critically on the hyperbolic picture. Without this picture, one could not use the implicit connectivity encoding (which depends on hyperbolic straightening), and would be forced to explicitly maintain the full connectivity of the common refinement, as done by Sun et al. [2015]. Likewise, our high-quality interpolation scheme (shown in Figure 3) relies on calculations in the light cone model of the hyperbolic plane.

\subsection{Other Methods}

Conformal Mapping. Other methods for conformal parameterization do not provide a general solution. For instance, early methods based on linear finite elements [Lévy et al. 2002; Desbrun et al. 2002; Mullen et al. 2008] do not guarantee injectivity, nor do they handle cone singularities. More recent linear methods support cones [BenChen et al. 2008; Vintescu et al. 2017; Sawhney and Crane 2017], but injectivity is still missing. Orbifold methods (e.g., [Aigerman and Lipman 2016]) provide injectivity, but support only a restricted set of cone configurations where cone angles cannot be prescribed. Anglebased methods [Sheffer et al. 2005] rely on nonconvex optimization, with no general convergence guarantees. Finally, Bobenko et al. [2015] and later Zhang et al. [2014] provide connections between discrete conformal equivalence and circle patterns.

Injective Mapping. Discrete uniformization has a special relationship to methods for locally injective mapping, since CETM is often used for initialization [Chien et al. 2016; Campen and Zorin 2017b; Campen et al. 2019]; we provide even stronger guarantees. Unlike [Mandad and Campen 2019; Shen et al. 2019] we do not claim to guarantee injectivity in floating point-yet still achieve injectivity in extremely challenging scenarios (Section 8.3.2). Overall we observe that the freedom to modify the triangulation during optimization leads to significantly improved robustness-see Section 8.3.

\section{PRELIMINARIES}

This section provides essential definitions needed to motivate and derive our algorithms; some readers may wish to skip ahead to Section 4 , and return here for reference. The most important concept is illustrated in Figure 14: any triangle mesh can be interpreted as both a Euclidean polyhedron (Section 3.2) and a decorated ideal polyhedron (Section 3.3), leading to a definition of conformal equivalence across different triangulations (Section 3.5). For further background, see Bobenko et al. [2015] and Springborn [2019].
Fig. 11. An edge or triangle in a $\Delta$-complex might not be uniquely determined by its vertices. Here, performing intrinsic edge flips on an octahedron yields two distinct edges between the same pair of vertices $i$ and $j$, and two triangles with the same vertices $i, j$, and $k$. The sphere depicts the abstract connectivity.

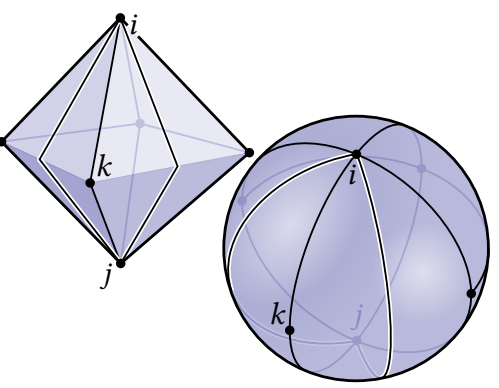

\subsection{Combinatorial Polyhedra}

Throughout we use $T=(\mathrm{V}, \mathrm{E}, \mathrm{F})$ to denote the connectivity of a manifold triangulation with vertices $V$, edges $E$, and faces $F$; we assume $T$ is orientable purely to simplify exposition. Even when the input is an ordinary (simplicial) triangulation, we may need to construct triangulations where, e.g., multiple edges connect the same two vertices, or two triangles share the same three vertices (Figure 11). Formally, we use triangulation to mean a $\Delta$-complex in the sense of Hatcher [2002, Section 2.1], which we encode via a halfedge data structure [Botsch et al. 2010, Section 2.3]. Though edges and faces are not uniquely determined by their vertices, for brevity we will still denote them by vertex pairs $i j \in \mathrm{E}$ and triples $i j k \in \mathrm{F}$, resp., where $i, j$, and $k$ need not be distinct. The notation $\phi_{i}^{j k}$ indicates a quantity $\phi$ at corner $i$ of a triangle $i j k$.

3.1.1 Combinatorial Edge Flip. For two triangles sharing a common edge, an edge flip replaces this edge with the opposite diagonal-we will need this operation in order to construct intrinsic Delaunay triangulations (Section 3.4). If we locally index the vertices of these two triangles as depicted in the inset figure, then the edge flip replaces the original triangles $i j k$ and $j i l$ with $j k l$ and $l k i$. Any other data stored on

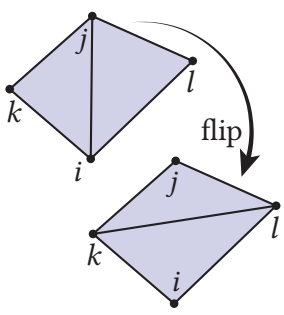
the triangulation must also be updated, as depicted in Figure 12 and discussed in Sections 3.2.1, 3.3.4, 5.1.1 and 5.2.1.

\subsection{Euclidean Polyhedra}

A Euclidean polyhedron is a surface that looks like the flat Euclidean plane everywhere except at a finite collection of cone points. The canonical example is an ordinary triangle mesh in $\mathbb{R}^{3}$, where the neighborhood around each vertex is isometric to a piece of a circular cone (see inset). For uniformization, however, we do not need to keep track of how the surface is embedded in space. Instead, we can store a purely intrinsic description of the geometry, given by the edge lengths $\ell: E \rightarrow \mathbb{R}_{>0}$ of a triangulation $T=(V, E, F)$. If these lengths satisfy the triangle inequalities in each triangle $i j k \in \mathrm{F}$,

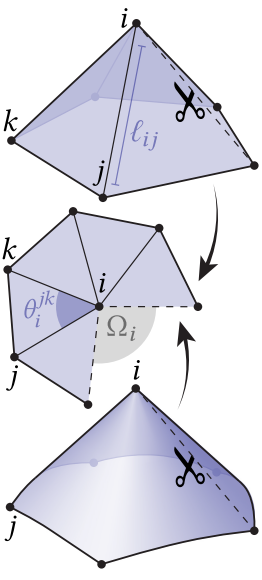
then we call $\ell$ a discrete metric. Other quantities such as corner 
angles $\theta_{i}^{j k}$ can be recovered from edge lengths via standard trigonometric formulas. In particular, each vertex $i \in \mathrm{V}$ has an angle defect $\Omega_{i}:=2 \pi-\sum_{i j k \in \mathrm{F}} \theta_{i}^{j k}$, which characterizes the flatness of the vertex, and is equal to the integral of Gaussian curvature over a small neighborhood around the cone point.

3.2.1 Euclidean Edge Flip. This description also enables us to change the triangulation of a Euclidean polyhedron without changing its intrinsic geometry. In particular, given only the edge lengths, the new edge length $\ell_{k l}$ resulting from an edge flip can be determined by laying out the known triangles $i j k$ and $j i l$ in the Euclidean plane, and measuring the distance between vertices $k$ and $l$. More robust numerical strategies are discussed by Fisher et al. [2007] and Sharp et al. [2019b].

3.2.2 Conformally Equivalent Edge Lengths. We say that two discrete metrics $\ell, \tilde{\ell}: E \rightarrow \mathbb{R}_{>0}$ on the same triangulation $\mathrm{T}=(\mathrm{V}, \mathrm{E}, \mathrm{F})$ are discretely conformally equivalent if at all edges $i j \in \mathrm{E}$

$$
\tilde{\ell}_{i j}=e^{\left(u_{i}+u_{j}\right) / 2} \ell_{i j}
$$

for some assignment of vertex scale factors $u: V \rightarrow \mathbb{R}$. These metrics are conformally equivalent if and only if they induce the same length cross ratios [Springborn et al. 2008, Section 2]

$$
\mathfrak{c}_{i j}=\frac{\ell_{i l} \ell_{j k}}{\ell_{l j} \ell_{k i}}
$$

We will give a definition of conformal equivalence for Euclidean polyhedra with different connectivity in Section 3.5.

\subsection{Hyperbolic Polyhedra}

3.3.1 Models of Hyperbolic Geometry. Just as the sphere $S^{2}$ is a surface of constant curvature $K=+1$, the hyperbolic plane $H^{2}$ is a surface of constant negative curvature $K=-1$. Unlike $S^{2}$, there is no way to smoothly embed $H^{2}$ in Euclidean $\mathbb{R}^{3}$ isometrically, i.e., without distorting its geometry [Hilbert 1901]. Instead, we must visualize it through one of several models, each of which faithfully represents only some of its geometric features. A good analogy is the Mercator projection of the globe, which preserves angles but

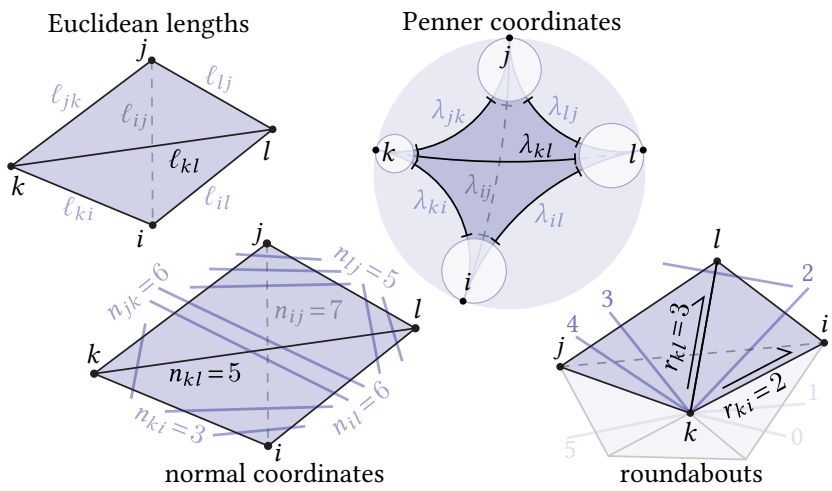

Fig. 12. For each edge flip, we need to update any data stored on edges. Here we indicate quantities involved in updating Euclidean edge lengths (top left), Penner coordinates (top right), normal coordinates (bottom left) and roundabouts (bottom right).

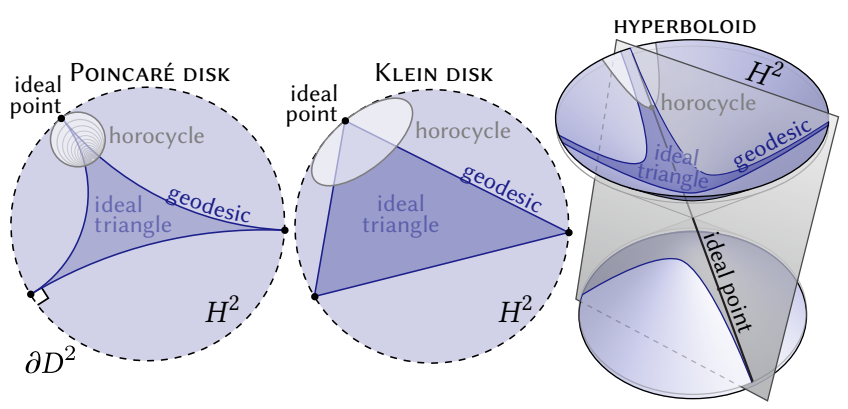

Fig. 13. Since the hyperbolic plane $H^{2}$ cannot be isometrically embedded in $\mathbb{R}^{3}$, it must be understood through the use of several "models"-here we illustrate how several key quantities are realized in each model.

distorts the size of land masses. Figure 13 depicts three models that are useful for our purposes. For further background on hyperbolic geometry, see Cannon et al. [1997]; Alekseevskij et al. [1993].

In the Poincaré disk model, points in $H^{2}$ are identified with points in the open unit disk $D^{2}:=\left\{p \in \mathbb{R}^{2}:|p|<1\right\}$. Although this disk looks like a finite piece of the Euclidean plane, lengths at a point $p \in D^{2}$ get scaled by $2 /\left(1-|p|^{2}\right)$ so that short distances near the boundary $\partial D^{2}$ represent large distances in $H^{2}$. One can hence travel any distance along a straightest curve or geodesic without ever reaching the boundary-limit points on $\partial D^{2}$ are called ideal points. Though geodesics are straight in $H^{2}$, in the Poincaré model they appear as circular arcs orthogonal to $\partial D^{2}$. The Poincaré model is conformal: angles between circular arcs give the true angle between geodesics in $H^{2}$. Finally, just as a straight line in $\mathbb{R}^{2}$ can be viewed as a circle of "infinite radius," a horocycle is the limit of a family of increasingly large circles tangent at a common point-drawn in the Poincaré model as a circle tangent to the boundary.

The Beltrami-Klein model is much like the Poincaré model, but with a different metric. Geodesics appear as straight lines, but Euclidean angles no longer give the true angles in $H^{2}$, i.e., the BeltramiKlein model is not conformal. Horocycles in the Beltrami-Klein model appear as ellipses. This model helps explain the relationship between Euclidean and hyperbolic polyhedra (Section 3.3.3).

The hyperboloid model represents $H^{2}$ as the upper sheet of the two-sheeted hyperboloid. Just as the sphere is the set of all points $p \in$ $\mathbb{R}^{3}$ such that $\langle p, p\rangle=1$, this hyperboloid is the set of all points satisfying $\langle p, p\rangle_{2,1}=-1$, where $\langle p, q\rangle_{2,1}:=p_{x} q_{x}+p_{y} q_{y}-p_{z} q_{z}$ is the Lorentz inner product; this inner product is also

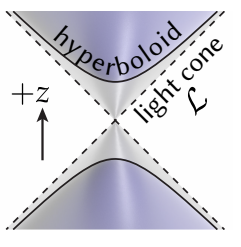
used to measure the angles and lengths of vectors tangent to the hyperboloid. Geodesics in $H^{2}$ correspond to intersections of the hyperboloid with planes through the origin, and ideal points are identified with lines in the light cone $\mathcal{L}:=\left\{p \in \mathbb{R}^{3}:\langle p, p\rangle_{2,1}=0\right\}$. Horocycles are obtained by taking a plane tangent to $\mathcal{L}$, shifting it in the positive $z$-direction, and intersecting with the hyperboloid. Thus, we can identify horocycles with points in the positive light cone $\mathcal{L}^{+}:=\left\{p \in \mathcal{L}: p_{z}>0\right\}$; each point $p \in \mathcal{L}^{+}$also corresponds to the plane $\left\{q \in \mathbb{R}^{3}:\langle p, q\rangle_{2,1}=-1\right\}$. The hyperboloid model is essential for developing our interpolation scheme-see Section 6.4. 


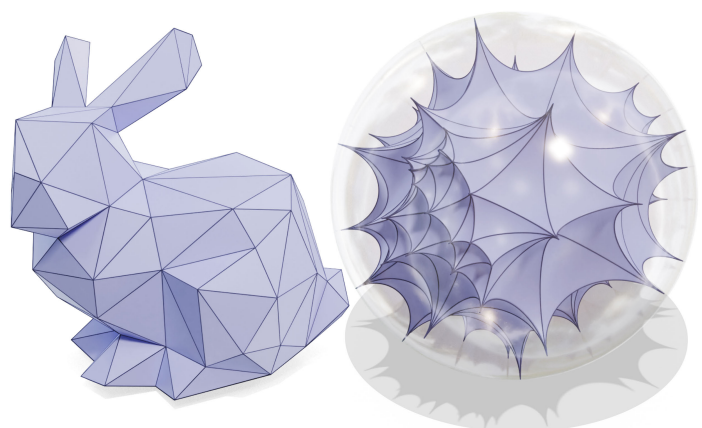

Fig. 14. An ordinary triangle mesh (left) can always be viewed as an ideal hyperbolic polyhedron (right), i.e., surface made from triangles of constant negative curvature and all three vertices at infinity.

3.3.2 Ideal Polyhedra. An ideal hyperbolic polyhedron is a surface of constant negative curvature, and a finite collection of cusps analogous to Euclidean cone points (Figure 14, right). We can construct ideal polyhedra by gluing together ideal triangles: regions of $\mathrm{H}^{2}$ bounded by three geodesics approaching three ideal points at infinity (Figure 13). A strange fact about ideal triangles is that they are all congruent, i.e., they are identical up to isometries of $H^{2}$. Hence, the geometry of an ideal polyhedron is determined entirely by how neighboring triangles $i j k$, jil are glued togethernamely, how far we slide them along the shared geodesic $i j$. One way to quantify gluings is to use shear coordinates, which for each edge $i j$ give the distance $Z_{i j} \in \mathbb{R}$ between the altitudes

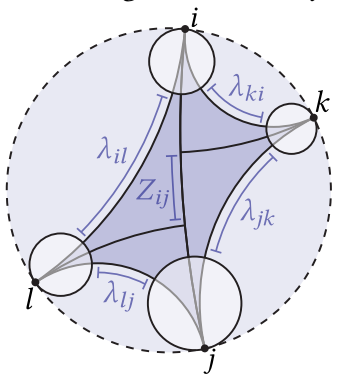
dropped from opposite vertices $k$ and $l$ (see inset). Alternatively, we can pick an arbitrary horocycle at each vertex, yielding a decorated ideal polyhedron. Though edges of an ideal triangle do not have finite length, there is now a finite distance $\lambda_{i j} \in \mathbb{R}$ between the horocycles at $i$ and $j-$ these values are called the Penner coordinates. Shear and Penner coordinates are related by

$$
Z_{i j}=\frac{1}{2}\left(\lambda_{i l}-\lambda_{l j}+\lambda_{j k}-\lambda_{k i}\right)
$$

(see [Penner 2012, Corollary 4.16, p. 40]). Note that if the horocycles at $i$ and $j$ overlap, $\lambda_{i j}$ will be negative. Yet unlike negative Euclidean lengths, negative Penner coordinates will cause no trouble for discrete uniformization. Likewise, whereas Euclidean lengths must satisfy the triangle inequality, any three Penner coordinates $\lambda_{i j}, \lambda_{j k}, \lambda_{k i} \in \mathbb{R}$ (whether positive or negative) can be realized by some choice of horocycles.

3.3.3 Euclidean-Ideal Correspondence. Every Euclidean polyhedron gives rise to an ideal polyhedron, in the following way. Any triangle $i j k \in \mathrm{F}$ drawn in its Euclidean circumdisk can be interpreted as an ideal triangle in the Beltrami-Klein model. To glue two ideal triangles $i j k, j i l$ together along an edge $i j$, we simply identify the same points as in the Euclidean polyhedron. An ideal polyhedron constructed this way will have shear coordinates $Z_{i j}=\log c_{i j}$, and if we assign Penner coordinates

$$
\lambda_{i j}=2 \log \ell_{i j}
$$

we get a decorated version of the same polyhedron. In general, we can move from Euclidean to hyperbolic polyhedra by "taking a logarithm"-for example, Equation 5 now just becomes the logarithm of Equation 4. More importantly, for a fixed triangulation, a conformal scaling of edge lengths $a$ la Equation 3 corresponds to a shift in horocycles of the form

$$
\tilde{\lambda}_{i j}=\lambda_{i j}+u_{i}+u_{j}
$$

In other words, conformally equivalent edge lengths $\ell, \tilde{\ell}$ describe the same ideal polyhedron, just decorated with different horocycles.

3.3.4 Ptolemy Flip. Penner coordinates are easily updated during edge flips via Ptolemy's relation [Penner 2012, Corollary 4.16, p. 40]. Letting $\ell_{i j}=e^{\lambda_{i j} / 2}$ for each edge in Figure 12 (top right), we compute

$$
\ell_{k l}=\left(\ell_{k i} \ell_{l j}+\ell_{j k} \ell_{l i}\right) / \ell_{i j} .
$$

The new Penner coordinate is then $\lambda_{k l}=2 \log \left(\ell_{k l}\right)$ (Figure 12, top right). Since Equation 8 is a rational expression in $\ell$, it is often simplest to just store and manipulate the edge lengths $\ell$ rather than the Penner coordinates $\lambda$. See Section 8.1 for further discussion of numerics.

Importantly, this so-called Ptolemy flip is the same as a Euclidean edge flip if and only if the two Euclidean triangles are concyclic (Figure 9). In general, Euclidean flips may distort the discrete conformal structure even though they preserve the Euclidean geometry (Figure 15), whereas Ptolemy flips always preserve the hyperbolic metric, hence the conformal structure. Moreover, Euclidean flips are well-defined only when the triangle inequalities are satisfied, whereas Ptolemy flips are always well-defined.

\subsection{Delaunay Triangulations}

For polyhedral surfaces, discrete conformal equivalence is defined in terms of Delaunay triangulations-not because they are "nice" in a numerical sense, but because they are key to establishing the discrete uniformization theorem mentioned in Section 1. Delaunay triangulations have similar but distinct definitions in the Euclidean and ideal hyperbolic settings.

3.4.1 Intrinsic Delaunay Triangulations. A planar triangulation is Delaunay if there are no vertices on the interior of any triangle circumcircle. Equivalently, we can ask that every interior edge $i j$ contained in triangles $i j k$, jil satisfy the local Delaunay condition

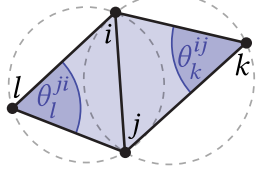

$$
\theta_{k}^{i j}+\theta_{l}^{j i} \leq \pi
$$

This characterization generalizes to Euclidean polyhedra, since the edge lengths $\ell$ are sufficient to determine the angles $\theta$. Such intrinsic Delaunay triangulations can be found using a simple greedy algorithm: while any edge fails to satisfy Equation 9, perform a Euclidean flip (à la Section 3.2.1). This algorithm terminates after finitely many flips [Indermitte et al. 2001; Bobenko and Springborn 2007], and in practice takes about $|\mathrm{E}|$ flips on real-world meshes [Sharp et al. $2019 \mathrm{~b}$, Figure 10]. Note if two triangles are inscribed in a common circle, then either diagonal satisfies Equation 9 (Figure 9).

3.4.2 Ideal Delaunay Triangulations. A hyperbolic analogue is an ideal Delaunay triangulation [Springborn 2019, Section 4]: if $\ell=e^{\lambda / 2}$ 


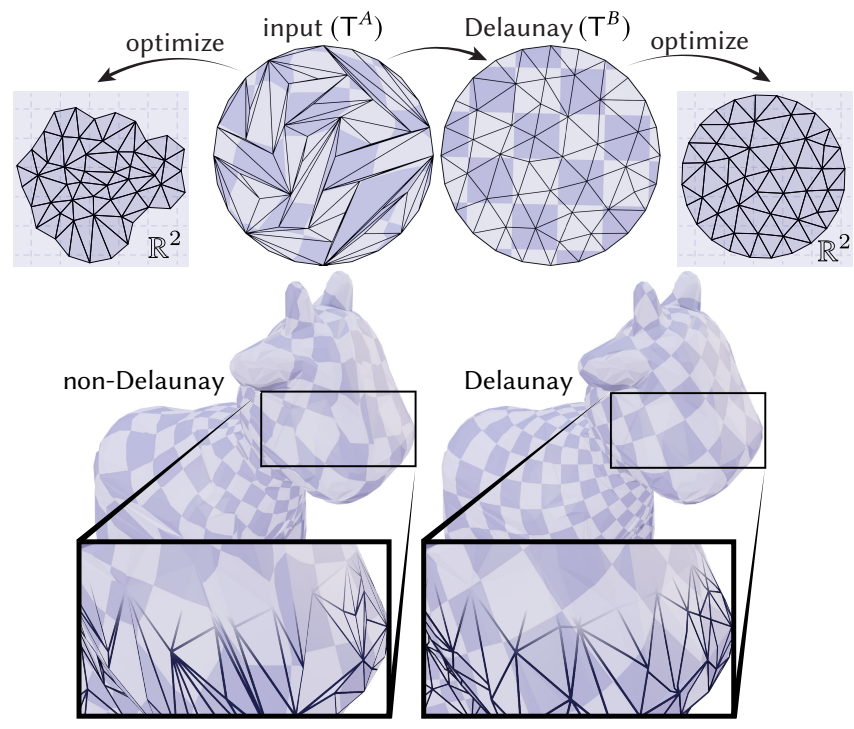

Fig. 15. Top: uniformization should leave a flat region unchanged, but unless one first flips to an intrinsic Delaunay triangulation, Ptolemy flips performed during optimization will distort the given shape. Bottom: in general, flipping to intrinsic Delaunay first tends to yield a better map.

are edge lengths associated with given Penner coordinates $\lambda$, then every edge must satisfy the local ideal Delaunay condition

$$
\ell_{i j}^{2}\left(\ell_{j k} \ell_{k i}+\ell_{i l} \ell_{l j}\right)<\left(\ell_{i l} \ell_{k i}+\ell_{j k} \ell_{l j}\right)\left(\ell_{i l} \ell_{j k}+\ell_{k i} \ell_{l j}\right)
$$

which we obtain by combining Equations 3 and 10 from Springborn [2019]. We can again find such a triangulation by greedily flipping edges, but this time using Ptolemy flips. Remarkably, if Equation 10 is satisfied globally, then the lengths $\ell$ always describe a valid $E u-$ clidean intrinsic Delaunay triangulation [Springborn 2019, 4.14]. Yet working in the ideal setting enables us to start with lengths that do not describe a valid Euclidean metric and flip to a valid one (Figure 8).

\subsection{Discrete Conformal Equivalence}

We can now state what it means for polyhedra with different triangulations to be discretely conformally equivalent. Consider in particular two Euclidean polyhedra with the same vertex set $\mathrm{V}$, encoded as intrinsic Delaunay triangulations $(\mathrm{T}, \ell)$ and $(\tilde{\mathrm{T}}, \tilde{\ell})$. Two mathematically equivalent definitions provide not only different geometric perspectives, but also lead to different algorithms.

Euclidean perspective. One definition of discrete conformal equivalence is that there must exist a sequence of Euclidean intrinsic Delaunay triangulations

$$
(\mathrm{T}, \ell)=\left(\mathrm{T}_{1}, \ell_{1}\right), \ldots,\left(\mathrm{T}_{n}, \ell_{n}\right)=(\tilde{\mathrm{T}}, \tilde{\ell})
$$

where each consecutive pair $\left(\mathrm{T}_{i}, \ell_{i}\right),\left(\mathrm{T}_{i+1}, \ell_{i+1}\right)$ is related by either (i) a conformal scaling of edge lengths, à la Equation 3, or (ii) Euclidean edge flips of concyclic triangle pairs, à la Section 3.2.1.

Hyperbolic perspective. The other definition says that $(\mathrm{T}, \ell)$ and $(\tilde{\mathrm{T}}, \tilde{\ell})$ are discretely conformally equivalent if the associated ideal hyperbolic polyhedra (as defined in Section 3.3.3) are isometric, i.e., if they simply describe different triangulations of the same negatively-curved surface. Concretely, any modification of the initial Penner coordinates via Equation 7 followed by Ptolemy flips to an ideal Delaunay triangulation will yield a discretely conformally equivalent surface. This perspective is illustrated in Figure 8.

An important difference between these two perspectives is that in the Euclidean case one must stop to perform edge flips whenever the triangulation becomes non-Delaunay, whereas in the hyperbolic case scaling and flipping are decoupled: one can adjust Penner coordinates freely, and need not stop to perform flips.

\section{UNIFORMIZATION}

Here we describe our procedure for planar parameterization-see Section 7 for the spherical case. This procedure is outlined in Figure 2; detailed pseudocode can be found in the supplement.

Given an input mesh $\mathrm{T}^{A}$, we first flip to an intrinsic Delaunay triangulation $\mathrm{T}^{B}$ (à la Section 3.4.1), which preserves the Euclidean geometry and defines the discrete conformal structure. We then solve an optimization problem for scale factors $u$ that transform $\mathrm{T}^{B}$ into a triangulation $\mathrm{T}^{C}$ with the prescribed angle defects (Section 4.3). After optimization, we lay $\mathrm{T}^{C}$ out in the plane (Section 4.5). However, this layout does not yet provide a mapping of the input mesh to the plane-Sections 5 and 6 describe how to construct such a map. Note that if we skip the first step (i.e., do not flip to intrinsic Delaunay) then we could work with just two triangulations, and get a map that is still locally injective, but may exhibit conformal distortion (see Figures 15 and 24).

\subsection{Variational Formulation}

The input to our discrete uniformization procedure is the intrinsic Delaunay triangulation $T^{B}$, and target angle defects $\Omega^{*}: \mathrm{V} \rightarrow \mathbb{R}$ which must satisfy a discrete Gauss-Bonnet condition:

$$
\frac{1}{2 \pi} \sum_{i \in \mathrm{V}} \Omega_{i}^{*}=|\mathrm{V}|-\left|\mathrm{E}^{B}\right|+\left|\mathrm{F}^{B}\right|
$$

(see Section 4.4 for a generalization to surfaces with boundary). Note that target defects $\Omega_{i}^{*}$ must be smaller than $2 \pi$, since the sum of angles around a vertex is always positive. Minimizing a convex energy $\mathcal{E}$ then yields scale factors $u$ relative to $\mathrm{T}^{B}$.

Note that unlike CETM we flip to Delaunay whenever we need to evaluate the energy or its derivatives (as detailed in Section 4.2). This process is completely hidden inside a callback routine-from the perspective of the optimizer, one simply has to solve an unconstrained problem that is convex and twice continuously differentiable $\left(C^{2}\right)$.

\subsection{Energy Evaluation}

To evaluate our energy for any given $u$, we first compute the edge lengths $\tilde{\ell}_{i j}=e^{\left(u_{i}+u_{j}\right) / 2} \ell_{i j}^{B}$, and flip to the corresponding ideal Delaunay triangulation $\widetilde{T}=(\mathrm{V}, \widetilde{\mathrm{E}}, \widetilde{\mathrm{F}})$ using Ptolemy flips. These flips change the Euclidean geometry but preserve the discrete conformal structure. We will use $\tilde{\lambda}, \tilde{\theta}$, and $\widetilde{\Omega}$ to denote the corresponding Penner coordinates, interior angles, and angle defects, resp. 
4.2.1 Energy. The discrete conformal energy is then given by

$$
\mathcal{E}(u)=\sum_{i \in \mathrm{V}}\left(2 \pi-\Omega_{i}^{*}\right) u_{i}-\sum_{i j \in \widetilde{\mathrm{E}}} \pi \tilde{\lambda}_{i j}+\sum_{i j k \in \widetilde{\mathrm{F}}} 2 f\left(\tilde{\lambda}_{i j}, \tilde{\lambda}_{j k}, \tilde{\lambda}_{k i}\right),
$$

where $f\left(\tilde{\lambda}_{i j}, \tilde{\lambda}_{j k}, \tilde{\lambda}_{k i}\right)$ is given by

$$
\frac{1}{2}\left(\tilde{\theta}_{i}^{j k} \tilde{\lambda}_{j k}+\tilde{\theta}_{j}^{k i} \tilde{\lambda}_{k i}+\tilde{\theta}_{k}^{i j} \tilde{\lambda}_{i j}\right)+\pi\left(\tilde{\theta}_{i}^{j k}\right)+\pi\left(\tilde{\theta}_{j}^{k i}\right)+\pi\left(\tilde{\theta}_{k}^{i j}\right) .
$$

Here Л denotes Milnor's Lobachevsky function

$$
\pi(\theta):=-\int_{0}^{\theta} \log |2 \sin u| d u,
$$

which is related to Clausen's integral via $Л(\theta)=\frac{1}{2} \mathrm{Cl}_{2}(2 \theta)$; the latter is implemented in standard numerical packages [Galassi et al. 1994]. Unlike CETM, which extends the energy linearly to handle lengths that violate the triangle inequality, we always evaluate this energy on the intrinsic Delaunay triangulation implied by the current scale factors. Constant shifts relative to [Springborn et al. 2008, Equation 7] ensure that, when evaluated this way, the energy, its gradient, and its Hessian vary continuously with the log scale factors $u$-even though different scale factors can induce different triangulations [Springborn 2019, Proposition 7.12]. Note also that a Euclidean edge flip preserves this energy $\mathcal{E}$ if and only if the two participating triangles are concyclic-again motivating the use of Delaunay triangulations.

4.2.2 Gradient. At each vertex $i \in \mathrm{V}$, the gradient of the energy is

$$
\partial_{u_{i}} \mathcal{E}=\widetilde{\Omega}_{i}-\Omega_{i}^{*}
$$

Note, then, that any stationary point $\partial_{\mathcal{u}} \mathcal{E}=0$ achieves the desired angle defects $\widetilde{\Omega}=\Omega^{*}$.

4.2.3 Hessian. The Hessian is given by the positive-semidefinite cotan Laplacian $L \in$ $\mathbb{R}^{|\mathrm{V}| \times|\mathrm{V}|}[$ MacNeal 1949, Section 3.2]; [Crane et al. 2013a, Chapter 6]. Since a $\Delta$ complex may contain more than one edge with the

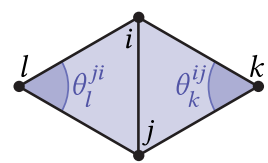
same endpoints (see for example Figure 11), the off-diagonal entries $L_{i j}$ and $L_{j i}$ are obtained by summing the values $\frac{1}{2}\left(\cot \theta_{k}^{i j}+\cot \theta_{l}^{j i}\right)$ over all edges $i j \in \widetilde{\mathrm{E}}$ with endpoints $i$ and $j$, where $k, l$ are the vertices opposite the edge. For each vertex $i \in \mathrm{V}$, we then have a diagonal entry $L_{i i}=-\sum_{i j \in \widetilde{\mathrm{E}}} L_{i j}$, where the sum is taken over all edges incident on $i$. Note that self-edges (where $i=j$ ) make no contribution.

\subsection{Optimization}

Since the energy $\mathcal{E}$ is convex and globally $C^{2}$, it can be minimized using any standard method for convex optimization. We use Newton's method with backtracking line search, as described in Algorithms 9.5 and 9.2 of Boyd and Vandenberghe [2004], resp. In particular, we use the descent direction $v \in \mathbb{R}^{|\mathrm{V}|}$ obtained by solving the linear system

$$
L v=\partial_{u} \mathcal{E}
$$

where $\partial_{u} \mathcal{E} \in \mathbb{R}^{|\mathrm{V}|}$ encodes the gradient defined in Section 4.2.2. Note that the matrix $L$ has a one-dimensional kernel of constant vectors. We simply use the solution $v$ that has no constant component (which corresponds to a global scaling). Although $L$ is rank deficient,

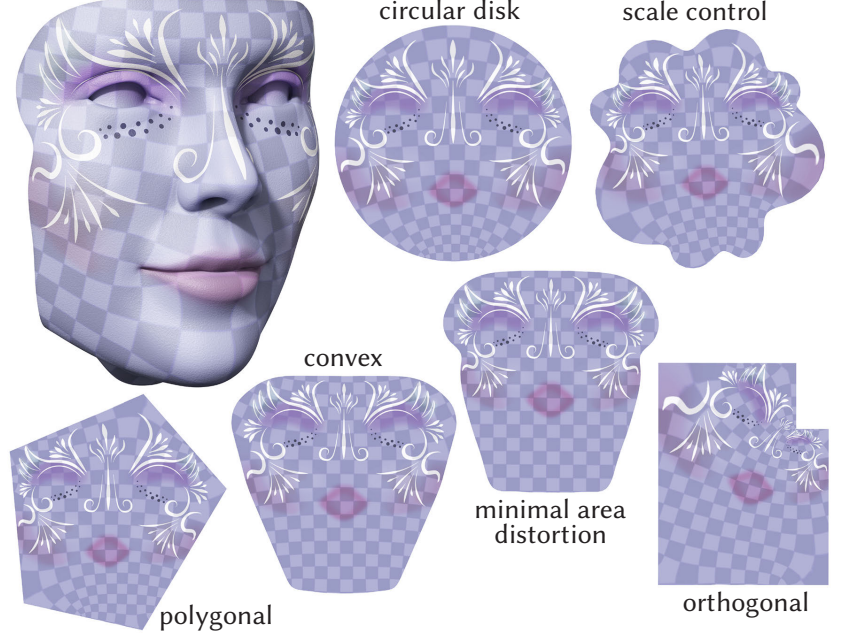

Fig. 16. Our algorithm guarantees existence of a locally injective discrete conformal map for any prescribed boundary lengths or angles, which can be used to achieve a rich variety of behavior. Spherical uniformization also provides a globally injective conformal map to the unit disk.

the system is solvable: Gauss-Bonnet ensures that the right-hand side sums to zero. We initialize Newton's method with $u=0$, but since the energy is convex this choice will not affect the result (apart from a global scale).

\subsection{Surfaces with Boundary}

For a smooth surface $M$ with boundary $\partial M$, the space of conformal maps to the plane is parameterized by a real-valued function along the boundary-geometrically, this function can be determined by prescribing either the scale factors $u$ or the curvature density $\kappa d s$ along $\partial M$ (see [Sawhney and Crane 2017, Section 4.2] for further discussion). We can specify such conditions by either a scale factor $u_{i}$ or target exterior angle $\kappa_{i}^{*}$ at each boundary vertex $i \in \partial \mathrm{V}$. To enforce these conditions, we glue together two copies of the input mesh along the boundary (as in Jin et al. [2004]), reducing the problem to the no-boundary case. Unlike CETM, we can hence always find a solution with the prescribed boundary data. Note that this construction extends Springborn [2019], which does not consider surfaces with boundary; Sun et al. [2015] describe a similar scheme in the case of prescribed boundary curvature. Maps to the circular disk are handled in a similar fashion, but using the spherical uniformization from Section 7.

4.4.1 Fixed Boundary Curvature. Suppose we want our flattened domain to have an exterior angle $\kappa_{i}^{*}$ at a boundary vertex $i$. The angle sum at $i$ must then be equal to $\pi-\kappa_{i}^{*}$, hence on the doubled domain we prescribe an angle defect $\Omega_{i}^{*}=2 \pi-$ $2\left(\pi-\kappa^{*}\right)=2 \kappa_{i}^{*}$. Since the solution is unique, it must be symmetric across the two copies of the original

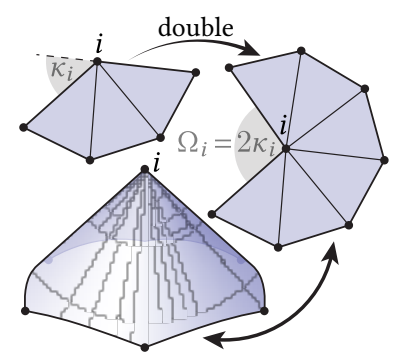


mesh. Hence, if we cut the uniformized surface along the original boundary curve, each half will exhibit the desired angles $\kappa^{*}$. The only requirement is that the angle defects and exterior angles satisfy a Gauss-Bonnet condition $\sum_{i \in \mathrm{V}} \Omega_{i}^{*}+\sum_{i \in \partial \mathrm{V}} \kappa_{i}^{*}=|\mathrm{V}|-|\mathrm{E}|+|\mathrm{F}|$. In Figure 16 we assign target angles that yield convex $\left(\kappa_{i}^{*}>0\right)$, orthogonal $\left(\kappa_{i}^{*} \in \frac{\pi}{2} \mathbb{Z}\right)$, or polygonal boundaries $\left(\kappa_{i}^{*}=0\right.$ almost everywhere).

4.4.2 Fixed Boundary Scale Factors. To prescribe boundary scale factors, we fix the values $u_{i}$ at vertices $i$ of the doubled domain corresponding to the original boundary. For instance, setting $u_{i}=0$ at all boundary vertices yields minimal area distortion [Chebyshev 1899 , p. 242] in the sense that it minimizes the variation in scale factors [Springborn et al. 2008, Appendix E]-see Figure 16. Fixing these values restricts the convex energy $\mathcal{E}$ to a linear subspace; hence we are still solving a convex problem. To compute the descent direction, we now solve the same system (Equation 12), except that we set zero Dirichlet boundary conditions at the boundary vertices, since we do not want these values to change. The minimizer will exhibit the target angle defects at interior vertices, since the gradient still only vanishes when $\widetilde{\Omega}=\Omega^{*}$.

\subsection{Planar Layout}

The final scale factors $u$ provide an intrinsic description of the flattened surface, which we then lay out in the plane. Just as we do during optimization, we first scale the edge lengths (à la Equation 3) and flip to Delaunay using Ptolemy edge flips to get a final triangulation $\left(\mathrm{T}^{C}, \ell^{C}\right)$. Since the final edge lengths $\ell^{C}$ describe a triangulation that is flat away from cone singularities (Figure 4), we can simply lay the triangles out in the plane one at a time to get a parameterization with no flipped triangles. (Section 8.2 discusses a numerically robust alternative.) Since coordinates are discontinuous across cuts, we store values $z_{i}^{j k} \in \mathbb{R}^{2}$ at corners.

\section{CORRESPONDENCE}

We now describe a data structure for tracking correspondence between different triangulations of the same polyhedron. In particular, we introduce an implicit, integer-based encoding that is easily updated via local formulas during each edge flip. An explicit geometric correspondence is later extracted from this information once all flips have been performed (e.g., after uniformization)-see Section 6. Since this encoding uses only integer data, it avoids robustness issues that can arise with floating-point representations (e.g., Figure 27).

Explicitly, to encode the correspondence between any two triangulations $T_{1}, T_{2}$ with the same vertex set $\mathrm{V}$, we store

- normal coordinates, which count the number of times $\mathrm{T}_{1}$ crosses each edge of $\mathrm{T}_{2}$ (Section 5.1), and

- roundabouts, which give the circular ordering of edges from both $\mathrm{T}_{1}$ and $\mathrm{T}_{2}$ around each vertex (Section 5.2).

Normal coordinates enable us to later trace geodesic segments from each vertex $i$ to all neighboring vertices $j$ in $T_{1}$, yielding curves along $\mathrm{T}_{2}$ (Section 6.1). Roundabouts provide the correspondence between these traced segments and logical edges of $T_{1}$. This latter data is needed because the two endpoints $i, j$ of a traced segment may not uniquely determine an edge (Figure 11).
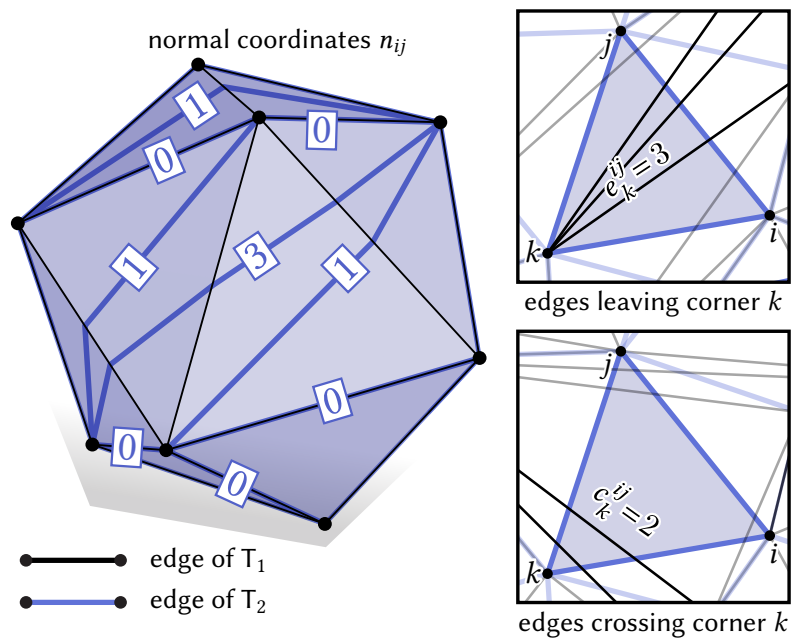

Fig. 17. Left: normal coordinates $n_{i j}$ count the number of times each edge $i j$ in a triangulation $\mathrm{T}_{2}$ crosses any edge of another triangulation $\mathrm{T}_{1}$ transversely. Right: these coordinates can be used to determine other quantities, such as how many edges of $T_{1}$ cross or leave a corner of a triangle from $T_{2}$.

For our flattening procedure we use this scheme to track the correspondence both between $\mathrm{T}^{A}$ and $\mathrm{T}^{B}$, and between $\mathrm{T}^{B}$ and $\mathrm{T}^{C}$ (see Figure 2). Note that in the remaining sections we use $\mathrm{H}$ to denote the halfedges associated with edges $\mathrm{E}$, i.e., the two possible orientations $\overrightarrow{i j} \neq \overrightarrow{j i}$ of each edge $i j$ in $\mathrm{E}$.

\subsection{Normal Coordinates}

Normal coordinates count the number of times a collection of curves cross each edge of a fixed triangulation (Figure 17). Our use of normal coordinates deviates from the standard treatment in two ways. First, rather than closed topological curves, we consider open geodesic segments that terminate at vertices. Second, we always assume that our normal coordinates encode the edges of another triangulation of the same vertex set. These assumptions enable us to develop a novel edge flip formula, given in Section 5.1.1. In particular, for each edge $i j$ of $\mathrm{T}_{2}$, we store the number of times $n_{i j} \in \mathbb{Z}_{\geq 0}$ that any edge of $\mathrm{T}_{1}$ crosses $i j$ transversely (Figure 17, left). Hence, for edges $i j$ shared by both $\mathrm{T}_{1}$ and $\mathrm{T}_{2}$ we have $n_{i j}=0$. From these numbers we can determine how many edges in $T_{1}$ emanate from corner $k$ of a triangle $i j k$ in $\mathrm{T}_{2}$ (excluding those along edges of $\mathrm{T}_{2}$ ):

$$
e_{k}^{i j}=\max \left(0, n_{i j}-n_{j k}-n_{k i}\right) .
$$

Likewise, the number of edges in $\mathrm{T}_{1}$ that cross corner $k$ of $i j k$ is

$$
c_{k}^{i j}=\frac{1}{2}\left(\max \left(0, n_{j k}+n_{k i}-n_{i j}\right)-e_{i}^{j k}-e_{j}^{k i}\right) .
$$

See Figure 17, right for examples.

5.1.1 Normal Coordinate Edge Flip. Consider two triangles $i j k$, $j i l$ from $\mathrm{T}_{2}$. In the simple case where no edge from $T_{1}$ terminates in a corner of either triangle (see inset), there is an edge flip update that resembles the Ptolemy relation [Mosher

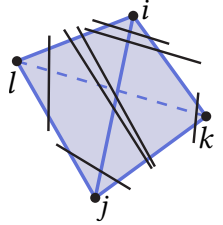


1988]; [Thurston and Yuan 2012, Equation 1]:

$$
n_{k l}=\max \left(n_{k i}+n_{l j}, n_{j k}+n_{l i}\right)-n_{i j} .
$$

In the general case, we must derive a more complicated formula (see supplement):

$$
\begin{array}{r}
n_{k l}=\max \left(0, c_{l}^{j i}+c_{k}^{i j}+\frac{1}{2}\left|c_{j}^{i l}-c_{j}^{k i}\right|+\frac{1}{2}\left|c_{i}^{l j}-c_{i}^{j k}\right|-\frac{1}{2} e_{l}^{j i}-\frac{1}{2} e_{k}^{i j}\right. \\
\left.+e_{i}^{l j}+e_{i}^{j k}+e_{j}^{i l}+e_{j}^{k i}+\delta_{n_{i j}}\right) .
\end{array}
$$

Here $\delta_{x}$ is the Kronecker delta, equal to 1 for $x=0$ and 0 otherwise.

\subsection{Roundabouts}

Although normal coordinates completely describe a triangulation sitting on top of $\mathrm{T}_{2}$, they do not tell us how the edges of this triangulation correspond to the edges of $\mathrm{T}_{1}$ since, as noted above, two endpoints may not uniquely identify an edge (Figure 11). We therefore augment our normal coordinates with what we call roundabouts, in analogy with roundabouts or traffic circles found on roadways. At each vertex $i \in \mathrm{V}$, these roundabouts describe how the outgoing halfedges of the two triangulations are interleaved.

More explicitly, for each halfedge $\overrightarrow{i j} \in \mathrm{H}_{2}$, the roundabout gives the first halfedge from $\mathrm{T}_{1}$ following $\overrightarrow{i j}$, encoded as an index $r_{i j} \in \mathbb{Z}_{\geq 0}$ (Figure 18). These indices start at zero, and enumerate the halfedges from $T_{1}$ in counter-clockwise order, starting at some arbitrary but fixed halfedge. Note that if a halfedge from $T_{2}$ coincides with a halfedge from $T_{1}$, the roundabout points to this halfedge, as indicated by self-arrows.

5.2.1 Roundabout Edge Flip. Using per-vertex indices (instead of a map from $H_{2}$ to $H_{1}$ ) reduces the edge flip update to integer arithmetic. In particular, to update roundabouts after flipping an edge $i j$ with opposite vertices $k, l$, we first update the normal coordinates as described in Section 5.1.1. We then have

$$
\begin{aligned}
& r_{\vec{k} l}=\bmod \left(r_{\overrightarrow{k i}}+e_{k}^{i l}+\delta_{n_{k i}}, \operatorname{deg}_{1}(k)\right), \\
& r_{\overrightarrow{l k}}=\bmod \left(r_{\overrightarrow{l j}}+e_{l}^{j k}+\delta_{n_{l j}}, \operatorname{deg}_{1}(l)\right),
\end{aligned}
$$

where $\operatorname{deg}_{1}(i)$ is the degree of vertex $i$ in the triangulation $\mathrm{T}_{1}$. In other words, to find the first outgoing halfedge of $\mathrm{T}_{1}$ following $\overrightarrow{k l} \in \mathrm{H}_{2}$, we start at $\overrightarrow{k i}$ and add the number of edges $e_{k}^{i l}$ of $\mathrm{T}_{1}$ that emanate from corner $k$ of triangle $k i l$. Also, if $\overrightarrow{k i}$ is coincident with a halfedge from

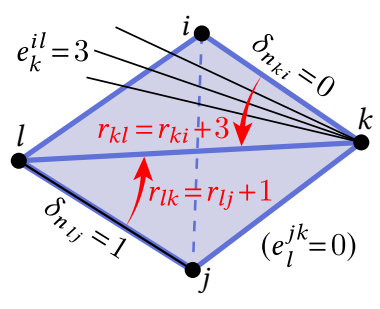
$\mathrm{T}_{1}$, we add 1 to advance past this halfedge. The mod operation accounts for wraparound. See inset for an example. This update resembles a combinatorial version of the signpost update from Sharp et al. [2019b, 3.2.1]: integer indices $r_{\overrightarrow{i j}}$ play the role of real-valued directions; the integer counts $e_{i}^{j k}$ play the role of real-valued angles.

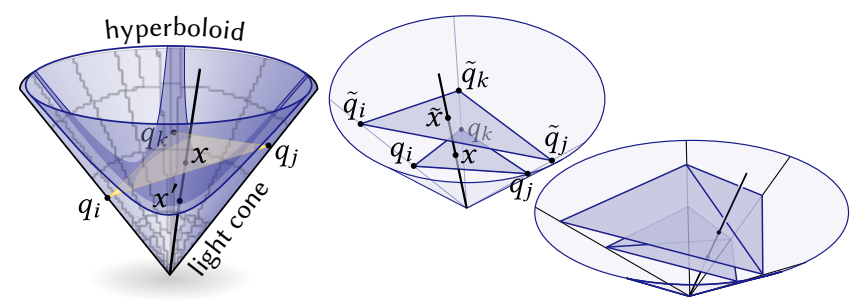

Fig. 19. By drawing triangles in the light cone (left), the map between surfaces can be found by drawing a straight line through the origin (center), which also works for two different triangulations (right).

\section{MAPPING}

Following uniformization (Section 4), we have three triangulations: the input $\mathrm{T}^{A}$ with vertex positions $f$, its intrinsic Delaunay triangulation $\mathrm{T}^{B}$, and the flattened mesh $\mathrm{T}^{C}$ with texture coordinates $z$ (Figure 2). For most tasks (e.g., texture mapping or remeshing), we will need an explicit map between $\mathrm{T}^{A}$ and $\mathrm{T}^{C}$, which we now construct. Using the correspondence data from Section 5 we first trace out geodesics to identify the points where edges of $\mathrm{T}^{A}$ and $\mathrm{T}^{C}$ intersect edges of $\mathrm{T}^{B}$ (Section 6.1). We then use these points to construct a common refinement $\mathrm{S}$, i.e., the smallest polygonal tessellation that contains all three triangulations (Section 6.3). Finally, we interpolate the functions $f$ and $z$ across $S$ (Section 6.4). The result is an ordinary polygon mesh with vertex coordinates $f_{i} \in \mathbb{R}^{3}$ and texture coordinates $z_{i}^{j k}$ at each triangle corner; these texture coordinates can be used for either piecewise projective or standard piecewise linear interpolation.

6.0.1 Layout in the Light Cone. As discussed in [Springborn et al. 2008, Section 3.4], conformally equivalent edge lengths naturally induce a piecewise projective map. However, when the triangulation is allowed to change, constructing this map becomes more difficult. A useful perspective, different from previous work [Bobenko et al. 2015; Sun et al. 2015; Springborn 2019], is to consider chordal triangles in the light cone-leading to simple interpolation formulas in homogeneous coordinates (e.g., Equation 16). We here give a brief sketch, which is made more precise in the supplement. In particular, take any Euclidean triangle and place it in $\mathbb{R}^{3}$ so that its vertices sit at points $q_{i}, q_{j}, q_{k}$ on the light cone (Figure 19, left). As discussed in Section 3.3, these points also define the vertices of a decorated ideal triangle. Hence, central projection from any point $x$ on the Euclidean triangle to the hyperboloid provides an explicit mapping between the Euclidean and ideal triangle. Moreover, if we

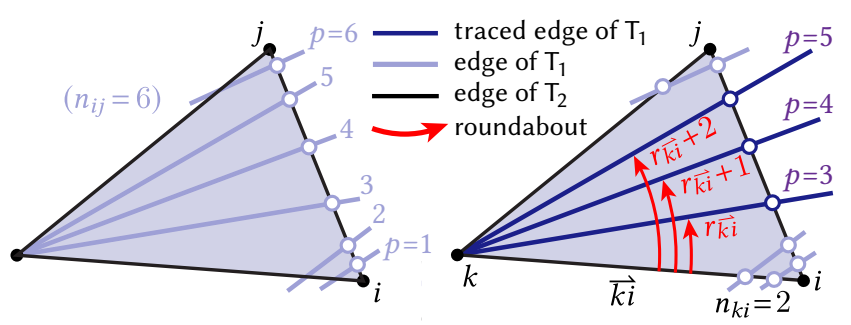

Fig. 20. Left: we index crossings along each halfedge $i j$ by an integer $p$. Right: for each halfedge we trace out curves leaving the opposite corner. 
apply a scaling $\tilde{q}_{i}=e^{u_{i}} q_{i}$ to each vertex, we get another chordal triangle; central projection between points $x$ and $\tilde{x}$ then gives the circumcircle-preserving projective map used for interpolation in Springborn et al. [2008] (Figure 19, center). The real power of this construction is that it enables us to map between different triangulations of the same vertices. Consider for instance two chordal triangles $i j k, j i l-i f$ we apply a flip and then scale the vertices $q$ as before, we get two chordal triangles $l k i, k l j$ sitting "above" the other ones (Figure 19, right). The map between these triangle pairs is still given by central projection, and can be expressed as a piecewise projective map on the four triangles of their common subdivision. This idea is extended in the supplement to general pairs of triangulations.

\subsection{Tracing Edges}

For the moment, consider just two triangulations $T_{1}, T_{2}$. We use the normal coordinates $n: E_{2} \rightarrow \mathbb{Z}_{\geq 0}$ to trace out the sequence of edges in $T_{2}$ crossed by each edge of $T_{1}$ (Section 6.1.1). The roundabouts $r: \mathrm{H}_{2} \rightarrow \mathbb{Z}_{\geq 0}$ uniquely identify each traced sequence with the appropriate element of $E_{1}$. To get the curve geometry, we lay out a triangle strip in the Euclidean or hyperbolic plane, and draw a straight line between endpoints (Section 6.2). The final curve is encoded by $1 \mathrm{D}$ barycentric coordinates $s, t \in[0,1]$ on each intersected edge. We enumerate points where edges of $\mathrm{T}_{1}$ cross a halfedge $\overrightarrow{i j}$ of $\mathrm{T}_{2}$ by a crossing index $p \in\left\{1, \ldots, n_{i j}\right\}$ (see Figure 20 , left).

6.1.1 Topological Tracing. To trace out all the edges of $\mathrm{T}_{1}$ over $\mathrm{T}_{2}$, we iterate over the halfedges $\overrightarrow{i j} \in \mathrm{H}_{2}$ and trace edges emanating from the opposite corner $k$ (Figure 20, right), namely, the edges with indices $p=1+n_{k i}, \ldots, 1+n_{k i}+e_{i j}$. This procedure is detailed in Algorithm 1. We identify the edge of $T_{1}$ corresponding to each traced curve $p$ by incrementing the roundabout $r \overrightarrow{k i}$ by $p-n_{k i}-1$. (By marking traced edges in $\mathrm{T}_{1}$, we avoid tracing edges twice.) Note that roundabouts must be used even for curves shared by both triangulations, since after a sequence of edge flips they may no longer correspond to the same logical edge.

The procedure NextEdge (Algorithm 2) uses the normal coordinates $n$ to determine the next halfedge $\overrightarrow{i j}$ and crossing index $p$ along the curve. The image below depicts the four possible cases-this pattern of edge crossings can be determined solely using the normal coordinates for the triangle $j i l$, via the formulas given in Section 5.1. See Appendix A.2 for further details.
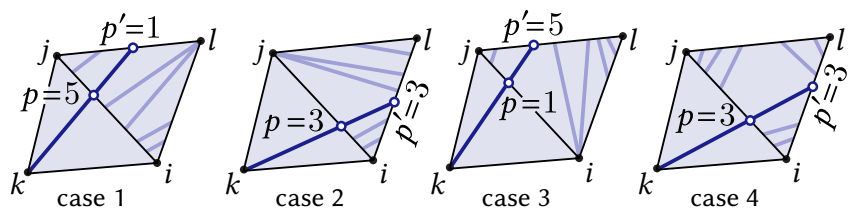

Note that the tracing procedure gives us each edge from $T_{1}$ as a sequence of edge crossings on $T_{2}$. To express the edges from $T_{2}$ as sequences of $\mathrm{T}_{1}$ edge crossings, we allocate an array of size $n_{i j}$ for each edge $i j \in \mathrm{E}_{2}$. Each time a traced edge $a b \in \mathrm{T}_{1}$ crosses $i j$, we store a reference to $a b$ in entry $p$ of the array (using roundabouts to get the edge index).

\subsection{Recovering Geodesics}

To get the geometry of each traced edge $a b \in$ $\mathrm{E}_{2}$, we use the crossing sequences computed in Section 6.1 and the edge lengths $\ell$ to incrementally lay out a triangle strip in the (Euclidean or hyperbolic) plane. We then intersect each interior edge $i j$ of this strip with the line from $a$ to $b$-by construction, this line will be contained entirely inside the strip. In particular, if $x_{i} \in \mathbb{R}^{2}$ are the vertices of a Euclidean triangle strip, we can solve the equation

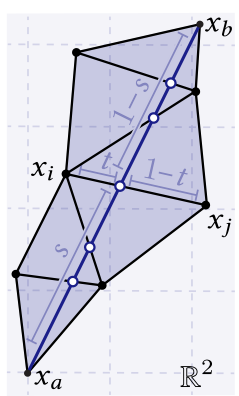

$$
(1-s) x_{a}+s x_{b}=(1-t) x_{i}+t x_{j}
$$

for the barycentric coordinates $s, t \in[0,1]$ of the intersection point. The hyperbolic case is conceptually the same except that we work in the hyperboloid model, and and also compute a scale factor $u$ at each intersection point-see Appendix A.3 for details.

\subsection{Common Refinement}

We now construct the common refinement $\mathrm{S}$ of $\mathrm{T}^{A}, \mathrm{~T}^{B}$, and $\mathrm{T}^{C}$. Here and in Appendix B we will refer to points along edges of $\mathrm{T}^{B}$ (resulting from tracing) as edge points, and any new vertices inserted into polygons as face points, reserving vertex for elements of $\mathrm{V}$. Likewise, an edge is the complete edge of some trian-

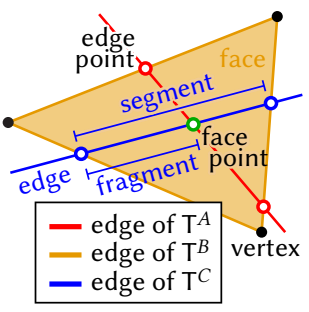
gulation, a segment is the restriction of an edge to a triangle, and a fragment is a piece of a segment (see inset).

6.3.1 Connectivity. First, for each edge $i j \in \mathrm{E}^{B}$, we use the procedures from Sections 6.1 and 6.2 to trace out (i) a Euclidean geodesic over $\mathrm{T}^{A}$ to obtain edge sequences and barycentric coordinates $(s, t)$, and (ii) a hyperbolic geodesic over $\mathrm{T}^{C}$ to obtain edge sequences, barycentric coordinates, and scale factors $u$. To determine the connectivity of $\mathrm{S}$ we slice up each triangle $i j k \in \mathrm{F}^{B}$ independently, via a strategy similar

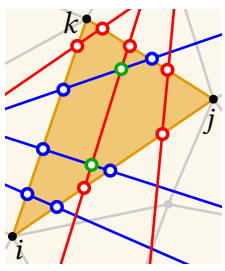
to Sharp et al. [2019b, Section 3.4]. To avoid computing segmentsegment intersections directly (which is not numerically robust), we devise a strategy that takes advantage of combinatorial information. Floating-point values serve only to determine the ordering of intersection points along edges-and since neighboring triangles have identical barycentric coordinates along their shared edge, we always obtain a consistent tessellation. See Appendix B for details.

\subsection{Interpolation}

The vertex coordinates $f_{i}$ and texture coordinates $z_{i}^{j k}$ define piecewise functions over the faces of $\mathrm{T}^{A}$ and $\mathrm{T}^{C}$, resp.; we now sample these functions onto $S$. To do so, we will also need the scale factors $u$ obtained while tracing hyperbolic geodesics. We again process each triangle $i j k \in \mathrm{T}^{B}$ independently. First, we interpolate data

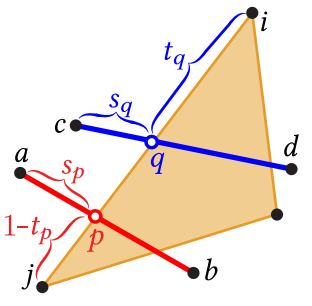


onto each edge $i j$ of the triangle. For each edge point $p$ along an edge $a b \in \mathrm{E}^{A}$, let $s_{p}, t_{p}$ be the barycentric coordinates along $a b$ and $i j$, resp.. Then $f_{p}=\left(1-s_{p}\right) f_{a}+s_{p} f_{b}$. Similarly, for an edge point $q$ along $c d \in \mathrm{E}^{C}$ we have homogeneous texture coordinates

$$
\hat{z}_{q}=e^{-u_{q}}\left(\left(1-s_{q}\right)\left(z_{c}, 1\right)+s_{q}\left(z_{d}, 1\right)\right),
$$

where $(z, 1)$ indicates that a 1 has been appended to $z$. The scale factors $e^{u}$ arise from projective rather than linear interpolationsee supplement for details. To get values of $f$ at edge points $q$, and values of $\hat{z}$ at edge points $p$, we linearly interpolate between adjacent known values along $i j$. Finally, to get the values at each face point, we write the endpoints of the two incident fragments in $2 \mathrm{D}$ barycentric coordinates relative to $i j k$, and compute the intersection point via Equation 19. The resulting $s, t$ values are then used to linearly interpolate $f$ and $\hat{z}$ from the segment endpoints. Note that since texture coordinates are discontinuous across cuts, we store $\hat{z}$ at corners rather than vertices. The final surface can be visualized by tessellating polygons into triangles; just as in [Springborn et al. 2008, Section 3.4] we perform a homogeneous divide on texture coordinates $\hat{z}$ at each sample point (e.g., each pixel).

\section{SPHERICAL UNIFORMIZATION}

We now consider conformal maps to the sphere $S^{2}$. Given a genus-0 Delaunay triangulation $\mathrm{T}=(\mathrm{V}, \mathrm{E}, \mathrm{F})$ with edge lengths $\ell: \mathrm{E}^{B} \rightarrow$ $\mathbb{R}_{>0}$, we give an algorithm that computes vertex positions $f: \mathrm{V} \rightarrow$ $S^{2} \subset \mathbb{R}^{3}$ that describe a discretely conformally equivalent convex sphere-inscribed polyhedron. The solution is guaranteed to exist, and is unique up to a Möbius transformation of the sphere.

The strategy used by CETM is essentially to delete the neighborhood of a special vertex $i^{*}$, conformally map this modified surface to a flat disk, and apply stereographic projection to the sphere, where the removed vertex $i^{*}$ is re-inserted. For a fixed triangulation, there are several problems. First, as discussed previously, a discretely conformally equivalent flat disk may not exist. Even if we allow the triangulation to vary, it is not immediately clear what to do about boundary edges (which cannot be flipped). Second, the final polyhedron may not be convex. In fact, many combinatorial triangulations do not admit any convex embedding in the sphere-conformal or otherwise [Rivin 1996]. Third, the map may become non-injective when vertex $i^{*}$ is re-inserted.

Imagine that we instead start with the object we want: a convex sphere-inscribed polyhedron $\mathcal{P}$ conformally equivalent to the input surface. If we stereographically project this polyhedron to the plane through any vertex $i^{*}$, we get a planar disk where all boundary vertices $j$ are connected to the same vertex $i^{*}$ at infinity (Figure 21, left). Stereographic projection preserves discrete conformal equivalence with the input, and since the polyhedron is convex, its stereographic projection will be a planar Delaunay triangulation [Brown 1979]and has hence a convex boundary. Hence, if we can construct such a triangulation, we can obtain the desired spherical conformal map (via stereographic projection).

To solve this problem, Springborn [2019] reformulates it in the hyperbolic setting where one can freely flip edges without invalidating the hyperbolic metric. Here, the Penner coordinates $\lambda_{i^{* j}}=2 \log \ell_{i^{*} j}$ incident on the special vertex $i^{*}$ are now infinite-effectively pushing the horocycle at $i^{*}$ off to infinity (Figure 21, right). This decorated
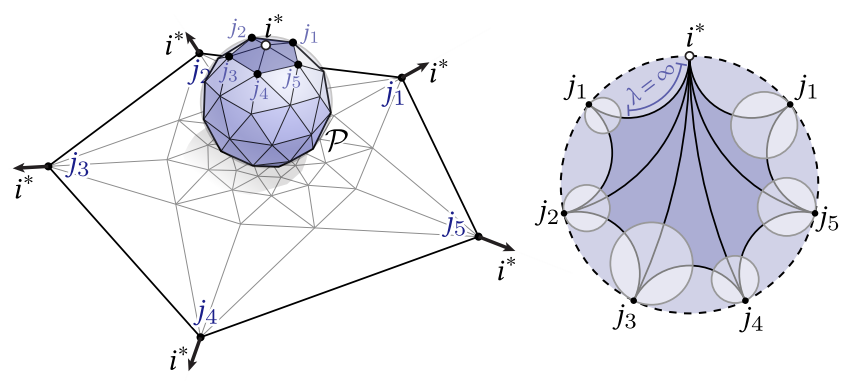

Fig. 21. Left: a convex polyhedron inscribed in the sphere can also be viewed, via stereographic projection, as a planar Delaunay triangulation with all boundary vertices connected to a vertex $i^{*}$ at infinity. Right: in the Poincaré model, the horocycle at $i^{*}$ shrinks to a point, and the incident Penner coordinates $\lambda_{i^{*} j}$ go to infinity.

polyhedron can be found via the same uniformization procedure as in Section 4, but with a few important modifications. For one, it uses a modified Delaunay flipping procedure which accounts for edges of infinite length (Section 7.1), and a modified energy which accounts for the boundary vertices $j$ adjacent to $i^{*}$ (Section 7.2). Linear inequality constraints on $u$ ensure that the edges $i^{*} j$ are convex and have the right cross ratios (Section 7.3). Solving a bounds-constrained optimization problem (Section 4.3) yields scale factors $u$ that describe the desired planar disk, which we can then stereographically project back onto the sphere.

\subsection{Modified Delaunay Flips}

Since some Penner coordinates are now infinite (Figure 21, right), we can no longer check the Delaunay condition using Equation 10. However, just as the Euclidean Delaunay condition is expressed in terms of angles (Equation 9), we can still express the ideal Delaunay condition

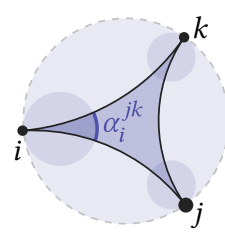
in terms of the arc length $\alpha_{i}^{j k}$ of the horocycle at vertex $i$ within triangle $i j k$ (see inset). Initially, all edge lengths $\ell$ are well-defined and we have

$$
\alpha_{i}^{j k}=\frac{\ell_{j k}}{\ell_{k i} \ell_{i j}} .
$$

Scaling lengths à la Equation 1 then gives new arc lengths

$$
\tilde{\alpha}_{i}^{j k}=e^{-u_{i}} \alpha_{i}^{j k}
$$

At the special vertex $i^{*}$, where $u_{i^{*}}=\infty$, we hence get $\tilde{\alpha}_{i}^{j k}=0$ as expected. An edge $i j$ then satisfies the ideal Delaunay condition if

$$
\tilde{\alpha}_{k}^{j i}+\tilde{\alpha}_{l}^{i j}<\tilde{\alpha}_{i}^{j k}+\tilde{\alpha}_{i}^{l j}+\tilde{\alpha}_{j}^{i k}+\tilde{\alpha}_{j}^{l i} .
$$

If this condition is not satisfied, we perform a Ptolemy flip (Equation 8). However, rather than compute $\tilde{\ell}_{k l}$ directly (which may be infinite), we first compute $\ell_{k l}$ via the Ptolemy relation and then scale to get $\tilde{\ell}_{k l}$. Importantly, if Equation 18 is satisfied with equality for an edge $k l$ opposite the special vertex $i^{*}$, we

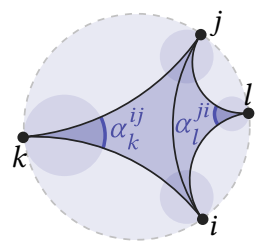
must pick the flip that connects $k l$ to $i^{*}$-since for any sequence 


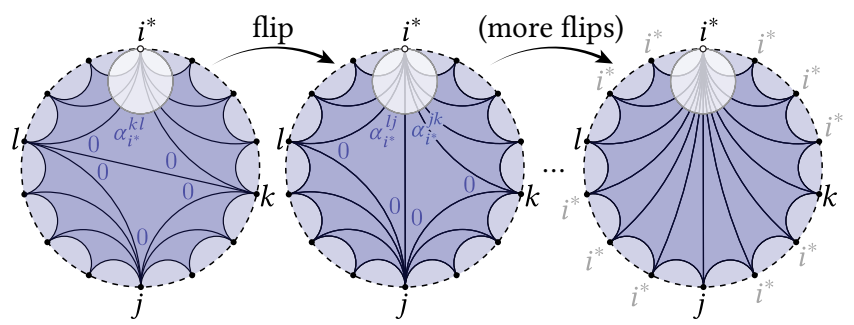

Fig. 23. To find a triangulation connecting the special vertex $i^{*}$ to all other vertices $j$, we put a finite horocycle at $i^{*}$ and send all other horocycles to infinity. Modified Delaunay flips then yield the desired triangulation.

of finite horocycles around $i^{*}$ approaching infinity, this is the edge that would belong to an ideal Delaunay triangulation.

\subsection{Spherical Variational Principle}

As in the Euclidean case, the energy and its derivatives are always evaluated on the triangulation $\widetilde{T}$ obtained by updating the Penner coordinates of $\mathrm{T}^{B}$ ( $\grave{a}$ la Equation 7) and flipping to an ideal Delaunay triangulation (à la Section 7.1). In particular, let $\mathrm{T}^{\circ}:=\left(\mathrm{V}^{\circ}, \mathrm{E}^{\circ}, \mathrm{F}^{\circ}\right)$ be the mesh obtained by removing the special vertex $i^{*}$ together with its incident edges and faces from $\widetilde{T}$. The energy for spherical uniformization is then

$$
\mathcal{E}_{S^{2}}(u)=2 \pi \sum_{i \in \mathrm{V}^{\circ}} u_{i}-\pi \sum_{i j \in \mathrm{E}^{\circ}} \tilde{\lambda}_{i j}+\sum_{i j k \in \mathrm{F}^{\circ}} 2 f\left(\tilde{\lambda}_{i j}, \tilde{\lambda}_{j k}, \tilde{\lambda}_{k i}\right)
$$

(see Springborn [2019, Equation 56 and Theorem 7.18], which differs by a constant that does not affect minimizers). For each vertex $i \in \mathrm{V}^{\circ}$, its gradient is

$$
\partial_{u_{j}} \mathcal{E}_{S^{2}}=\widetilde{\Omega}_{j}+\pi\left(\operatorname{deg}_{\mathrm{F}^{\circ}}(j)-\operatorname{deg}_{\mathrm{E}^{\circ}}(j)\right),
$$

where $\operatorname{deg}_{\mathrm{E}^{\circ}}(j)$ and $\operatorname{deg}_{\mathrm{F}^{\circ}}(j)$ are the number of edges and faces of $\mathrm{T}^{\circ}$ containing $j$, resp.; this degree difference will be -1 for vertices adjacent to $V^{\circ}$ (and zero otherwise). $\Omega^{*}$ does not appear because we do not consider cone singularities in the spherical case. The Hessian is again cotan-Laplace, where cotangents from any removed face are set to zero.

\subsection{Constraints}

In the fixed triangulation case, Bobenko et al. [2015, Proposition 3.2.1] observe that setting $u_{j}=-\lambda_{i^{*} j}$ ensures that the boundary edges $i^{*} j$ exhibit the right length cross ratio. However, in the variable triangulation case we do not know a priori which vertices $j$ will ultimately be adjacent to the removed vertex $i^{*}$ (since this set may change due to edge flips). Instead, as proposed by Springborn [2019], we impose the inequality con-

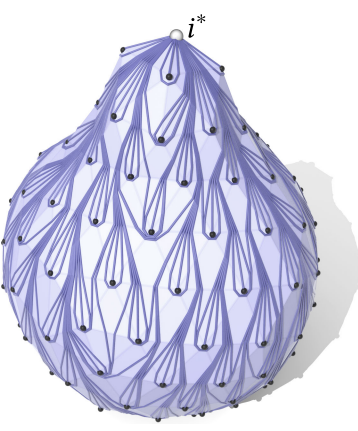

Fig. 22. Peacock triangulation. straint $u_{j} \geq-\lambda_{i^{*} j}$ for all vertices $j \in \mathrm{V}^{\circ}$, where $\lambda_{i^{*} j}$ is the geodesic distance between horocycles in the input triangulation. At a minimizer, these inequalities will be satisfied with equality for vertices $j$ adjacent to $i^{*}$.
To compute the geodesic distances, we first construct a triangulation that connects $i^{*}$ to all other vertices $j \in \mathrm{V}^{\circ}$ by minimal geodesics. To do so, we send all the horocycles except the one at $i^{*}$ to infinity-in the Poincaré model, the representative circles shrink down to points (Figure 23, left). In general, an edge connecting two vertices $j_{1}, j_{2} \neq i^{*}$ cannot be Delaunay, since the horocyclic arc length $\alpha$ at both vertices will be zero-hence smaller than the arc length of the complementary vertices (see Figure 23 and Equation 18). By flipping to a Delaunay triangulation, we ensure that any edge leaving a vertex $j \neq i^{*}$ connects only to $i^{*}$ (Figure 23, right). Moreover one can show that, due to the global Delaunay property, every such edge is a minimal geodesic [Springborn 2019, Proposition 5.16]. All other edges go from $i^{*}$ back to $i^{*}$, resulting in what we call a peacock triangulation (Figure 22). To get the values $\lambda_{i^{*} j}$, we then read off the distances between the original horocycles (rather than those that have been scaled down to points).

\subsection{Optimization}

Once we know $\lambda_{i^{*} j}$, we can solve the convex optimization problem

$$
\begin{aligned}
\min _{u: V^{\circ} \rightarrow \mathbb{R}} & \mathcal{E}_{S^{2}}(u) \\
\text { s.t. } & u_{j} \geq-\lambda_{i^{*} j}, \quad \forall j \in \mathrm{V}^{\circ} .
\end{aligned}
$$

This problem can be solved via a bounds-constrained Newton method; see Section 8.1 for further discussion.

\subsection{Spherical Layout}

After optimization, we have scale factors $u$ at vertices that describe a flat metric on the topological disk $\mathrm{T}^{\circ}$. We lay this disk out in the plane using the same procedure as described in Section 4.5, then stereographically project to get coordinates $z$ on the unit sphere $S^{2} \subset \mathbb{R}^{3}$ (re-inserting the special vertex $i^{*}$ at the center of stereographic projection). This final map is unique only up to Möbius transformations of the sphere; we compute a canonical Möbius transformation via Baden et al. [2018, Algorithm 1], using vertex rather than face areas to express the center of mass.

\subsection{Spherical Interpolation}

Interpolation is done as in Section 6, except we now lift coordinates $z \in \mathbb{R}^{3}$ to homogeneous coordinates $\hat{z} \in \mathbb{R}^{4}$, and scale factors must now account for both uniformization and stereographic projection. Let $\tilde{\ell}_{i j}$ be the edge lengths of $\mathcal{P}$, and $\ell_{i j}$ be the lengths from $\mathrm{T}^{B}$ after applying the same sequence of Ptolemy flips used for uniformization. Then solving Equation 3 within each triangle $i j k$ yields

$$
u_{i}=\log \left(\frac{\tilde{\ell}_{i j}}{\ell_{i j}} \frac{\ell_{j k}}{\tilde{\ell}_{j k}} \frac{\tilde{\ell}_{k i}}{\ell_{k i}}\right)
$$

(and similarly for $u_{j}, u_{k}$ ). Since stereographic projection preserves discrete conformal equivalence, these values agree across triangles. Also, since $\mathcal{P}$ is convex, normalizing interpolated coordinates gives an injective map to the unit sphere (for, e.g., texture mapping).

\section{EVALUATION}

This section evaluates the empirical behavior of our method, here referred to as conformal equivalence of polyhedral surfaces (CEPS). Our main points of comparison are the CETM algorithm of Springborn 

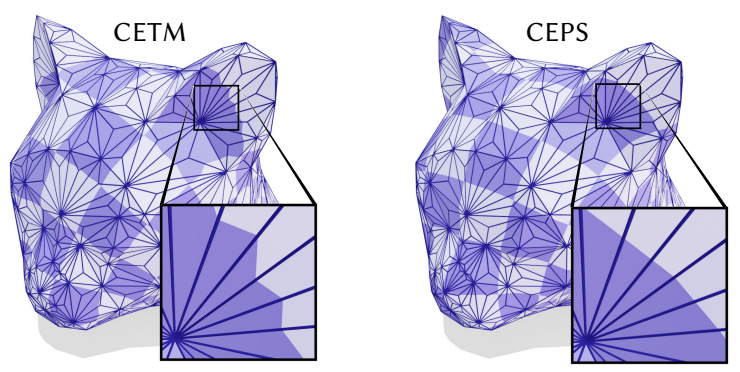

Fig. 24. Even when CETM succeeds, the quality of the map may be lower since it effectively considers a different notion of conformal equivalence (based on the input rather than Delaunay triangulation).

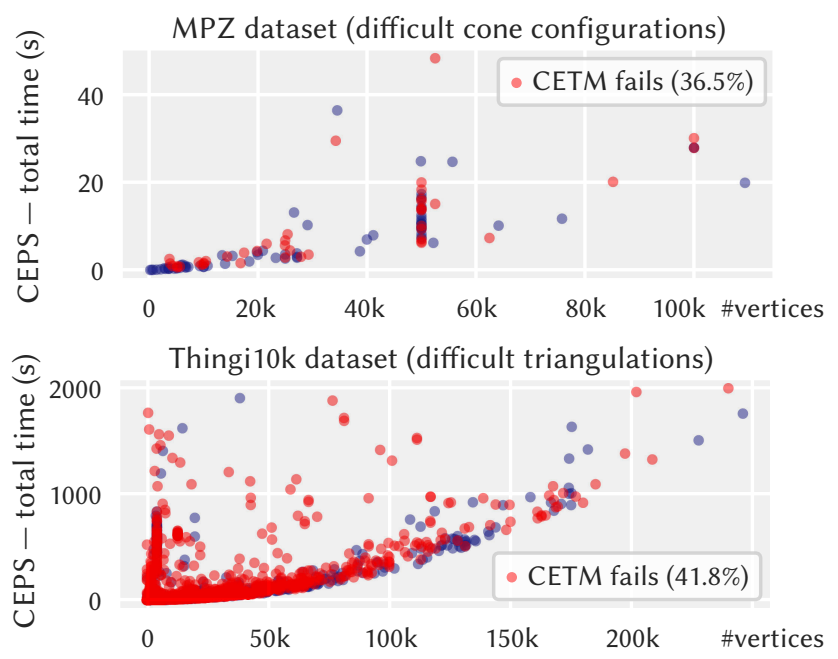

Fig. 25. Timings for our method (CEPS) on two datasets. Note that CETM fails on a large percentage of models where we succeed (highlighted in red).

et al. [2008], which does not use flips, and what we call uniformization with Euclidean edge flips (UEF) [Sun et al. 2015], which stops to flip concylic triangle pairs, as described in Section 2.1.2. All methods use identical code for tracking correspondence, à la Section 5 . We also briefly consider other methods for spherical conformal mapping (Section 8.3.3) and non-conformal injective mapping (Section 8.3.1)

The overall observation is that CEPS succeeds on far more models than CETM, and exhibits better scaling than UEF. Even when CETM does succeed, it may not provide as good of an approximation of a smooth conformal map (Figure 24). Moreover, our UEF implementation is more efficient than the one suggested by Sun et al. [2015] since it uses the implicit tracking scheme from Section 5 (which depends critically on the hyperbolic perspective), rather than an explicit overlay à la Fisher et al. [2007].

\subsection{Implementation}

Algorithms were implemented in double precision in $\mathrm{C}++$ using the halfedge implementation in GeometryCentral [Sharp et al. 2019a]. For cone flattening, we used Newton's method with backtracking line search [Boyd and Vandenberghe 2004, Algorithms 9.2 and 9.5],
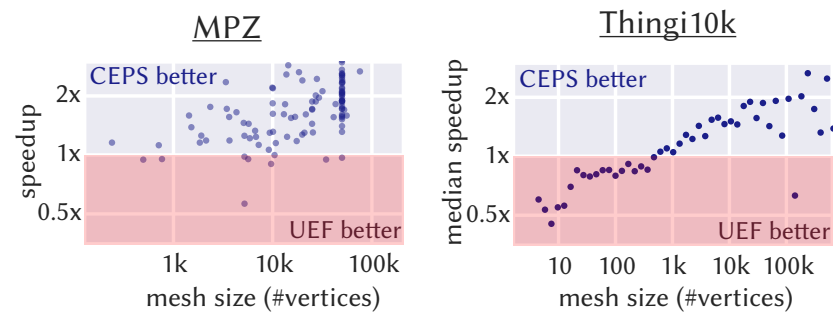

Fig. 26. Speedup of our method over UEF (total wall-clock time for computing scale factors). Beyond about $1 \mathrm{k}$ vertices, CEPS is typically faster.
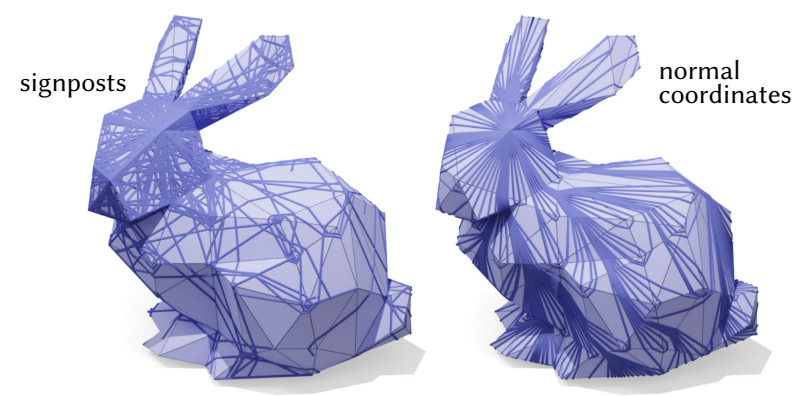

Fig. 27. Left: The signpost data structure fails to be numerically robust in extreme situations, such as when tracing the "peacock triangulation." Right: our integer-based encoding ensures we get the right connectivity.

using CHOLMOD to solve linear systems [Chen et al. 2008]. For spherical uniformization, we used bounds-constrained Newton's method with backtracking line search [Balay et al. 2019, 1997; Munson et al. 2014]. In practice, we use the implementation found in the PETsc/TAO library-specifically, we use the TAOBNLS solver [Benson et al. 2003, Section 4.2.1]. Timings were measured on an Intel i9-9980XE with 32 GB of RAM, using a single thread.

\subsection{Numerics}

Our algorithms are guaranteed to work in exact arithmetic for any valid input-in a real implementation we must also be careful about floating point error. Here we describe several useful techniques, though of course other improvements may be possible. Note that we mollify input edge lengths as described by Sharp and Crane [2020] (using $\delta=10^{-12}$ ) which helps with near-degenerate models and otherwise leaves the input untouched.

8.2.1 Euclidean Uniformization. One way to evaluate the intrinsic Delaunay condition (Equation 9) is to use the angle cotangents; Fisher et al. [2007] provide details. We instead check the hyperbolic condition (Equation 10)-even when constructing the Euclidean Delaunay triangulation-since it yields the same triangulation, but only involves rational arithmetic on edge lengths. When triangles are nearly concyclic, this condition may be violated (or satisfied) both before and after the edge flip, due to floating point error. Hence, we check Equation 10 for all edges, and perform a flip only if it increases the difference between right- and left-hand side.

8.2.2 Spherical Uniformization. For most models the choice of special vertex $i^{*}$ makes no difference, but on models with long, thin 


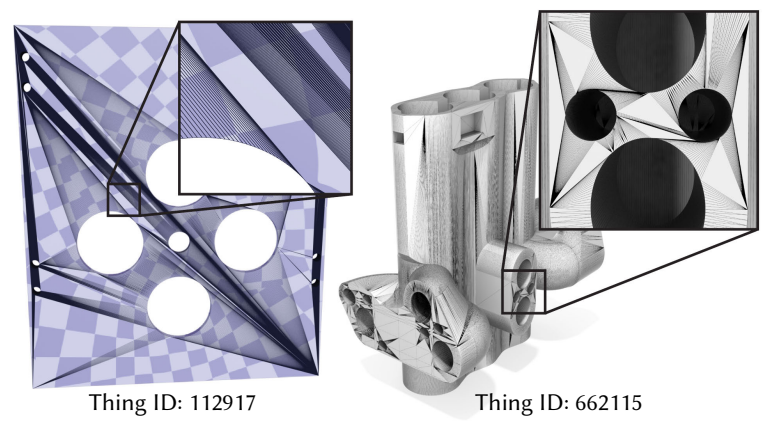

Fig. 28. Our implementation robustly handles extremely poor triangulations (left) failing only on the most pathological inputs (right). See Figure 1 for more examples.

features a useful heuristic is to put the vertex $i^{*}$ at the intrinsic median Sharp et al. [2019c, 8.3]; in practice we use the findCenter method from GeometryCentral [Sharp et al. 2019a]. When constructing the peacock triangulation in Section 7.3, values of $\ell$ can become large enough to result in floating point overflow. We therefore work instead with the values $\lambda=2 \log \ell$, which occupy a much smaller range. In particular, to compute the values $\alpha_{i}^{j k}$, we evaluate the log of Equation 17: $\log \alpha_{i}^{j k}=\frac{1}{2}\left(\lambda_{j k}-\lambda_{k i}-\lambda_{i j}\right)$, then exponentiate, and multiply by the scale factor $e^{-u_{i}}$ (which equals zero for all vertices but $i^{*}$ ). As before, Equation 18 gives the Delaunay condition in terms of $\alpha$. We also use the log-sum-exp trick [Blanchard et al. 2019, Equation 1.3] to help with numerical precision when applying the Ptolemy relation $\ell_{k l}=\left(\ell_{k i} \ell_{l j}+\ell_{j k} \ell_{l i}\right) / \ell_{i j}$. This means we write the log of the numerator as

$$
\frac{1}{2} \lambda_{k i}+\frac{1}{2} \lambda_{l j}+\log \left(1+e^{\left(\lambda_{j k} / 2+\lambda_{l i} / 2\right)-\left(\lambda_{k i} / 2+\lambda_{l j} / 2\right)}\right),
$$

where, without loss of generality, we label the vertices so that $\lambda_{k i}+$ $\lambda_{l j}>\lambda_{j k}+\lambda_{l i}$. We then subtract $\frac{1}{2} \lambda_{i j}$ to get the final log length for the new edge $k l$. (When building the peacock triangulation, we never explicitly compute the values of $\ell$.)

Layout. One could lay out triangles incrementally, as in [Springborn et al. 2008, Section 3.3]. We found it more robust to use the spectral layout of Mullen et al. [2008], which we use in all examples. This algorithm requires only the cotan-Laplace and mass matrices, which can be built directly from the final edge lengths $\ell^{C}$. Since lengths describe a flat metric, spectral layout incurs no further distortion.

Mapping. When laying out triangle strips (Section 6.2), we found that it improves floating point robustness to first incrementally compute the angles for each halfedge, and then use these angles to recover final vertex positions $x \in \mathbb{R}^{2}$. In meshes with near-degenerate triangles, we find that the hyperbolic layout procedure can sometimes fail to place points on the light cone due to floating-point errors. In particular, the new vertex coordinates $q_{l}$ might be at the origin, or contain NaNs. In this case, we replace the global strip layout with a local iterative straightening procedure (akin to nonlinear Gauss-Seidel). In particular, we consider two consecutive triangles at a time and update the location where the geodesic intersects the common edge-see supplement for details.

\subsection{Experiments}

8.3.1 Difficult Cone Configurations. We ran our method on the standard benchmark of Myles et al. [2014], referred to as $M P Z$, which contains challenging cone configurations. CEPS succeeds on all 114 models, including extraction of the common refinement. Maps were discretely conformal up to floating point error, with an average length cross ratio error of about $10^{-9}$, and no worse than about $10^{-4}$. In contrast, CETM succeeded on only 73 models (Figure 25, top) and was less than $2 \mathrm{x}$ faster (Figure 29, top). Moreover, the tracing and refinement steps of CEPS could be trivially parallelized over edges and faces, resp. UEF also succeeds on these models, but is generally slower than CEPS (Figure 26, left).

Many injective but non-conformal methods do not do as well on this difficult benchmark: as reported by Bright et al.

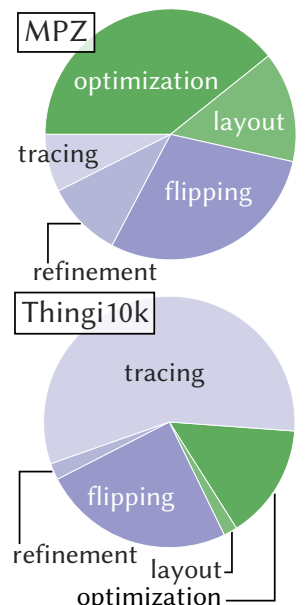

Fig. 29. Average breakdown of costs in CEPS; green tasks are shared by CETM.
[2017, Section 8.1], their method and the methods of Chien et al. [2016], Aigerman et al. [2014], Levi and Zorin [2014], and Lipman [2012] succeed on 104, 102, 97, 93, and 90 models, resp. Many of these methods have running times on the order of minutes or (on the most difficult examples) hours, versus seconds for our method. On the other hand, we must change/refine the triangulation, whereas these methods keep the triangulation fixed. Like CEPS, the combinatorial method of Zhou et al. [2020] succeeds on all MPZ models, but can yield highly distorted maps that are expensive to optimize; cost is again on the order of minutes to hours.

8.3.2 Difficult Triangulations. As a stress test of floating-point behavior, we parameterized all manifold meshes from Thingi10k, splitting disconnected meshes into their connected components $(32,744$ examples in total), and using a time out of 2000 seconds. Note that previous work on cone parameterization does not even attempt this benchmark, which has dramatically worse element quality than MPZ. For these examples we apply the greedy cone placement strategy from Springborn et al. [2008, Section 5.1], stopping when all log scale factors $u_{i}$ are in the range $[-5,5]$ (i.e., a max scale factor of about 150). Here CEPS successfully computes a parameterized mesh $\mathrm{S}$ for $98.6 \%$ of models, yielding an injective map on $97.7 \%$. Examples where we fail are quite pathological (e.g., Figure 28, right). Overall about $68 \%$ and $15 \%$ of failures were due to failure of iterative straightening or optimization (resp.) to converge within the time limit, and

Fig. 30. Since we allow edge flips, we need not worry how coarse the mesh is near large cones. Here we set all but one angle defect to almost $2 \pi$-the remaining vertex has an angle defect of -1032.79 .

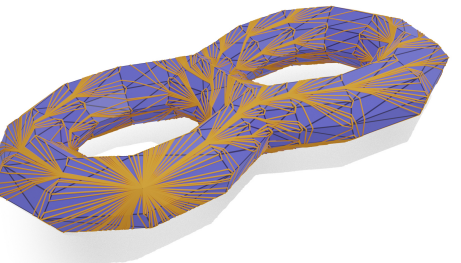




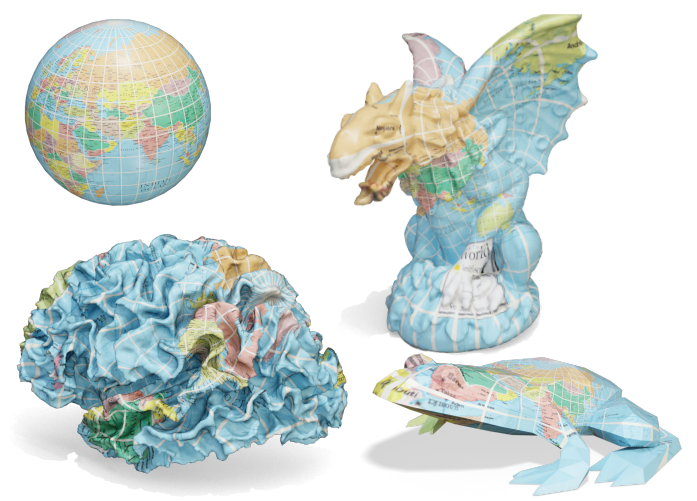

Fig. 32. In the genus-0 case, our method guarantees a bijective discrete conformal map to a convex polyhedron with vertices on the sphere.

for about $13 \%$ Delaunay flipping failed due to floating point error. The worst cross ratio error was typically around $10^{-5}$. CETM fails on almost half of these examples (Figure 25), and performance of UEF suffers as models get larger (Figure 26, right).

\subsubsection{Spherical Conformal Parameterization.} We ran our spherical algorithm on two other datasets: the Spherical Demon brain scan dataset of Yeo et al. [2009], and the anatomical surface dataset of Boyer et al. [2011] (Figure 32). On the brain dataset, where each model has about $230 \mathrm{k}$ faces, we obtained injective discrete conformal maps to the sphere on all 78 brain hemispheres, taking an average of 493 seconds per hemisphere. The anatomical surface models are topological disks, so we compute conformal maps to a

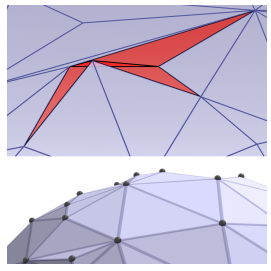

Fig. 31. Past spherical methods often exhibit foldover, and cannot guarantee convexity.

hemisphere. We succeed in computing these maps on all 187 of the manifold meshes without handles in the dataset. One of our maps contains degenerate triangles, but none have flipped triangles. The models take an average of 14.4 seconds to uniformize.

Past methods for spherical conformal parameterization largely compute maps to the sphere that are harmonic with respect to piecewise linear Dirichlet energy [Gu et al. 2004]. However, unless the input mesh is already Delaunay, such maps can have flipped triangles (Figure 31, right). More fundamentally, it is impossible for any method that uses a fixed triangulation to guarantee convexity-no matter what algorithm is used, or where the vertices are placedsince not all combinatorial triangulations admit a convex embedding in the sphere [Rivin 1996]. In practice, flipped triangles and nonconvex edges are quite common in discrete harmonic maps: on the brain dataset we observed, on average, foldover at about 100 edges and nonconvexity at about 20k edges when using the method of Kazhdan et al. [2012] (see inset). Other techniques for spherical conformal mapping gave very similar results [Crane et al. 2013b; Dym et al. 2019].

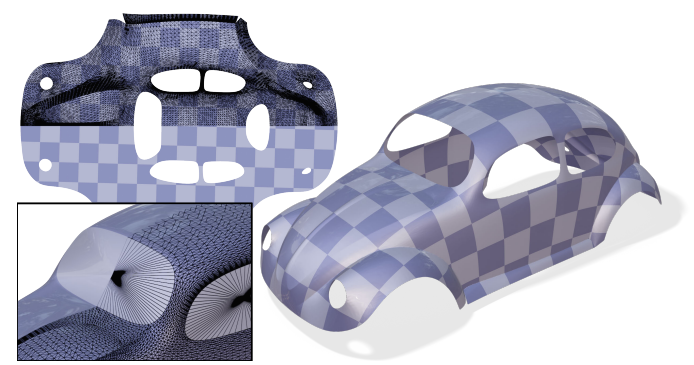

Fig. 33. We can handle multiply-connected domains by simply triangulating holes prior to flattening, then removing these triangles after flattening.

\subsection{Multiply-Connected Surfaces}

Many surfaces encountered in practice will have multiple boundary components. Though uniformization can be used to find a flat metric on such surfaces, this metric cannot always be laid out in the plane without additional cuts, due to nontrivial monodromy around boundary cycles (see for instance Hefetz et al. [2019, Figure 6]). Extension of discrete uniformization to multiply-connected circle domains and slit domains has been considered by Bobenko et al. [2016], but there is an even simpler alternative appropriate for practical texture mapping: just triangulate each of the holes, then remove these triangles after parameterization. Figure 33 shows one such example. A natural question is how to more directly control boundary behavior by setting the intrinsic lengths of inserted edges.

\section{LIMITATIONS AND FUTURE WORK}

The ability to modify the input triangulation is ultimately what enables one to establish a discrete uniformization theorem where existence is always guaranteed. From a practical point of view, it comes at a small cost: the output mesh has different connectivity than the input. Importantly, however, the common refinement S can be stored as a standard mesh with ordinary vertex and texture coordinates that can be used downstream; our implementation also outputs a sparse $\left|\mathrm{V}^{S}\right| \times\left|\mathrm{V}^{A}\right|$ matrix that interpolates values from the input mesh to the larger set of vertices in $S$. This refinement is also around 1.5-3x bigger than the input on common models (such as those in MPZ), and around $5 \mathrm{x}$ on most of Thingi10k-though on pathological models even just initial Delaunay flipping can blow up size by $45 \mathrm{x}$ or more. To reduce the final mesh size, it may be possible to "un-flip" some of the edges in the planar layout (as done by Kharevych et al. [2006, Section 5]), which incurs some conformal distortion but preserves injectivity. If one cares purely about injectivity, the initial Delaunay retriangulation step can also be skipped. The method is guaranteed to work only in exact arithmetic-it is natural to consider numerical improvements, or ways to further reduce dependence on floating-point calculations (e.g., during mesh extraction). We also did not consider uniformization over hyperbolic domains, though this case is well-supported by the same theory-see Springborn [2019] for further discussion. 


\section{ACKNOWLEDGMENTS}

This work was supported by a Packard Fellowship, NSF Award 1717320, DFG TRR 109, an NSF Graduate Research Fellowship, and gifts from Autodesk, Adobe, and Facebook.

\section{REFERENCES}

William Abikoff. 1981. The Uniformization Theorem. The American Mathematical Monthly 88, 8 (1981)

Ian Agol, Joel Hass, and William Thurston. 2006. The Computational Complexity of Knot Genus and Spanning Area. Trans. Amer. Math. Soc. 358, 9 (2006).

Noam Aigerman and Yaron Lipman. 2016. Hyperbolic Orbifold Tutte Embeddings. ACM Transactions on Graphics 35, 6 (2016).

Noam Aigerman, Roi Poranne, and Yaron Lipman. 2014. Lifted Bijections for Low Distortion Surface Mappings. ACM Transactions on Graphics 33, 4 (2014).

DV Alekseevskij, Eh B Vinberg, and AS Solodovnikov. 1993. Geometry of Spaces of Constant Curvature. In Geometry II. Springer.

Alex Baden, Keenan Crane, and Misha Kazhdan. 2018. Möbius Registration. Computer Graphics Forum 37, 5 (2018).

Satish Balay, Shrirang Abhyankar, Mark F. Adams, Jed Brown, Peter Brune, Kris Buschelman, Lisandro Dalcin, Alp Dener, Victor Eijkhout, William D. Gropp, Dmitry Karpeyev, Dinesh Kaushik, Matthew G. Knepley, Dave A. May, Lois Curfman McInnes, Richard Tran Mills, Todd Munson, Karl Rupp, Patrick Sanan, Barry F. Smith, Stefano Zampini, Hong Zhang, and Hong Zhang. 2019. PETSc Users Manual. Technical Report ANL-95/11 - Revision 3.11. Argonne National Laboratory.

Satish Balay, William D. Gropp, Lois Curfman McInnes, and Barry F. Smith. 1997. Efficient Management of Parallelism in Object Oriented Numerical Software Libraries. In Modern Software Tools in Scientific Computing, E. Arge, A. M. Bruaset, and H. P. Langtangen (Eds.). Birkhäuser Press.

Mirela Ben-Chen, Craig Gotsman, and Guy Bunin. 2008. Conformal Flattening by Curvature Prescription and Metric Scaling. In Computer Graphics Forum, Vol. 27.

Steve Benson, Lois Curfman McInnes, Jorge J More, and Jason Sarich. 2003. TAO Users Manual. Technical Report. Argonne National Lab., IL (US).

Pierre Blanchard, Desmond J Higham, and Nicholas J Higham. 2019. Accurate Computation of the Log-Sum-Exp and Softmax Functions. arXiv preprint (2019).

Alexander I Bobenko, Ulrich Pinkall, and Boris A Springborn. 2015. Discrete Conformal Maps and Ideal Hyperbolic Polyhedra. Geometry \& Topology 19, 4 (2015).

Alexander I Bobenko, Stefan Sechelmann, and Boris Springborn. 2016. Discrete Conformal Maps: Boundary Value Problems, Circle Domains, Fuchsian and Schottky Uniformization. In Advances in Discrete Differential Geometry. Springer.

Alexander I Bobenko and Boris A Springborn. 2007. A Discrete Laplace-Beltrami Operator for Simplicial Surfaces. Disc. \& Comp. Geom. 38, 4 (2007).

Mario Botsch, Leif Kobbelt, Mark Pauly, Pierre Alliez, and Bruno Lévy. 2010. Polygon Mesh Processing. CRC press.

Stephen Boyd and Lieven Vandenberghe. 2004. Convex Optimization. Cambridge university press.

Doug M Boyer, Yaron Lipman, Elizabeth St Clair, Jesus Puente, Biren A Patel, Thomas Funkhouser, Jukka Jernvall, and Ingrid Daubechies. 2011. Algorithms to Automatically Quantify the Geometric Similarity of Anatomical Surfaces. Proceedings of the National Academy of Sciences 108, 45 (2011).

Alon Bright, Edward Chien, and Ofir Weber. 2017. Harmonic Global Parametrization with Rational Holonomy. ACM Transactions on Graphics 36, 4 (2017).

Kevin Q Brown. 1979. Voronoi Diagrams from Convex Hulls. Information processing letters 9, 5 (1979).

Ulrike Bücking. 2016. Approximation of Conformal Mappings Using Conformally Equivalent Triangular Lattices. In Adv. in Disc. Diff. Geom. Springer.

Ulrike Bücking. 2018. $C^{\infty}$-Convergence of Conformal Mappings for Conformally Equivalent Triangular Lattices. Results in Mathematics 73, 2 (2018).

Marcel Campen, Ryan Capouellez, Hanxiao Shen, Leyi Zhu, Daniele Panozzo, and Denis Zorin. 2021. Efficient and Robust Discrete Conformal Equivalence with Boundary. (2021). arXiv:cs.CG/2104.04614

Marcel Campen, Hanxiao Shen, Jiaran Zhou, and Denis Zorin. 2019. Seamless Parametrization with Arbitrary Cones for Arbitrary Genus. ACM Transactions on Graphics 39, 1 (2019).

Marcel Campen and Denis Zorin. 2017a. On Discrete Conformal Seamless Similarity Maps. arXiv preprint (2017).

Marcel Campen and Denis Zorin. 2017b. Similarity Maps and Field-Guided T-Splines: A Perfect Couple. ACM Transactions on Graphics 36, 4 (2017).

James W Cannon, William J Floyd, Richard Kenyon, Walter R Parry, et al. 1997. Hyperbolic Geometry. Flavors of geometry 31 (1997).

Pafnutiǔ L'vovich Chebyshev. 1899. Euvres de P.L. Tchebychef. Vol. 1. Commissionaires de l'Académie Impériale des Sciences.

Wei Chen, Min Zhang, Na Lei, and Xianfeng David Gu. 2016. Dynamic Unified Surface Ricci Flow. Geom., Img. and Com. 3, 1 (2016).
Yanqing Chen, Timothy A Davis, William W Hager, and Sivasankaran Rajamanickam. 2008. Algorithm 887: CHOLMOD, Supernodal Sparse Cholesky Factorization and Update/Downdate. ACM Transactions on Mathematical Software (TOMS) 35, 3 (2008).

Edward Chien, Zohar Levi, and Ofir Weber. 2016. Bounded Distortion Parametrization in the Space of Metrics. ACM Transactions on Graphics 35, 6 (2016).

Keenan Crane. 2020. An Excursion through Discrete Differential Geometry. Proceedings of Symposia in Applied Mathematics, Vol. 76. American Mathematical Society, Chapter Conformal Geometry of Simplicial Surfaces.

Keenan Crane, Fernando de Goes, Mathieu Desbrun, and Peter Schröder. 2013a. Digital Geometry Processing with Discrete Exterior Calculus. In ACM SIGGRAPH 2013 Courses (SIGGRAPH '13). ACM, New York, NY, USA, Article 7.

Keenan Crane, Ulrich Pinkall, and Peter Schröder. 2013b. Robust Fairing via Conformal Curvature Flow. ACM Trans. Graph. 32, 4 (2013).

Mathieu Desbrun, Mark Meyer, and Pierre Alliez. 2002. Intrinsic Parameterizations of Surface Meshes. In Computer Graphics Forum, Vol. 21.

Nadav Dym, Raz Slutsky, and Yaron Lipman. 2019. Linear Variational Principle for Riemann Mappings and Discrete Conformality. Proceedings of the National Academy of Sciences 116, 3 (2019).

Jeff Erickson and Amir Nayyeri. 2013. Tracing Compressed Curves in Triangulated Surfaces. Discrete \& Computational Geometry 49, 4 (2013).

François Fillastre. 2008. Polyhedral Hyperbolic Metrics on Surfaces. Geometriae Dedicata 134, 1 (2008).

Matthew Fisher, Boris Springborn, Peter Schröder, and Alexander I Bobenko. 2007. An Algorithm for the Construction of Intrinsic Delaunay Triangulations with Applications to Digital Geometry Processing. Computing 81, 2-3 (2007).

Mark Galassi, Jim Davies, James Theiler, Brian Gough, Gerard Jungman, Patrick Alken, Michael Booth, Fabrice Rossi, and Rhys Ulerich. 1994. GNU Scientific Library. Vol. 20. ACM New York, NY, USA.

Xianfeng David Gu, Feng Luo, Jian Sun, and Tianqi Wu. 2018a. A Discrete Uniformization Theorem for Polyhedral Surfaces. Journal of Differential Geometry 109, 2 (2018).

Xianfeng David Gu, Ren Guo, Feng Luo, Jian Sun, and Tianqi Wu. 2018b. A Discrete Uniformization Theorem for Polyhedral Surfaces II. Journal of Differential Geometry 109, 3 (2018)

Xianfeng David Gu, Feng Luo, and Tianqi Wu. 2019. Convergence of Discrete Conformal Geometry and Computation of Uniformization Maps. Asian fournal of Mathematics 23, 1 (2019).

Xianfeng David Gu, Feng Luo, and Shing-Tung Yau. 2020. Computational Conformal Geometry behind Modern Technologies. Notices of the American Mathematical Society 67, 10 (2020).

Xianfeng David Gu, Yalin Wang, Tony F Chan, Paul M Thompson, and Shing-Tung Yau. 2004. Genus Zero Surface Conformal Mapping and Its Application to Brain Surface Mapping. IEEE transactions on medical imaging 23, 8 (2004).

Wolfgang Haken. 1961. Theorie Der Normalflächen: Ein Isotopiekriterium Für Den Kreisknoten. Acta Mathematica 105, 3-4 (1961).

A. Hatcher. 2002. Algebraic Topology. Cambridge University Press.

Eden Fedida Hefetz, Edward Chien, and Ofir Weber. 2019. A Subspace Method for Fast Locally Injective Harmonic Mapping. In Computer Graphics Forum, Vol. 38.

David Hilbert. 1901. Ueber Flächen von Constanter Gaussscher Krümmung. Trans. Amer. Math. Soc. 2,1 (1901).

Claude Indermitte, Th M Liebling, Marc Troyanov, and Heinz Clémençon. 2001. Voronoi Diagrams on Piecewise Flat Surfaces and an Application to Biological Growth. Theoretical Computer Science 263, ARTICLE (2001).

Miao Jin, Yalin Wang, Shing-Tung Yau, and Xianfeng David Gu. 2004. Optimal Global Conformal Surface Parameterization. In IEEE Visualization 2004.

Michael Kazhdan, Jake Solomon, and Mirela Ben-Chen. 2012. Can Mean-Curvature Flow Be Modified to Be Non-Singular?. In Computer Graphics Forum, Vol. 31.

Liliya Kharevych, Boris Springborn, and Peter Schröder. 2006. Discrete Conformal Mappings via Circle Patterns. ACM Transactions on Graphics 25, 2 (2006).

Hellmuth Kneser. 1929. Geschlossene Flächen in Dreidimensionalen Mannigfaltigkeiten. Jahresbericht der Deutschen Mathematiker-Vereinigung 38 (1929).

Patrice Koehl and Joel Hass. 2015. Landmark-Free Geometric Methods in Biological Shape Analysis. Journal of The Royal Society Interface 12, 113 (2015).

Mina Konaković, Keenan Crane, Bailin Deng, Sofien Bouaziz, Daniel Piker, and Mark Pauly. 2016. Beyond Developable: Computational Design and Fabrication with Auxetic Materials. ACM Trans. Graph. 35, 4 (2016).

Zohar Levi and Denis Zorin. 2014. Strict Minimizers for Geometric Optimization. ACM Transactions on Graphics 33, 6 (2014).

Bruno Lévy, Sylvain Petitjean, Nicolas Ray, and Jérome Maillot. 2002. Least Squares Conformal Maps for Automatic Texture Atlas Generation. ACM Transactions on Graphics 21, 3 (2002).

Yaron Lipman. 2012. Bounded Distortion Mapping Spaces for Triangular Meshes. ACM Transactions on Graphics 31, 4 (2012).

Yaron Lipman and Ingrid Daubechies. 2011. Conformal Wasserstein Distances: Comparing Surfaces in Polynomial Time. Advances in Mathematics 227, 3 (2011). 
Feng Luo. 2004. Combinatorial Yamabe Flow on Surfaces. Communications in Contemporary Mathematics 6, 5 (2004).

Richard H MacNeal. 1949. The Solution of Partial Differential Equations by Means of Electrical Networks. Ph.D. Dissertation. California Institute of Technology.

Manish Mandad and Marcel Campen. 2019. Exact Constraint Satisfaction for Truly Seamless Parametrization. In Computer Graphics Forum, Vol. 38.

Haggai Maron, Meirav Galun, Noam Aigerman, Miri Trope, Nadav Dym, Ersin Yumer Vladimir G Kim, and Yaron Lipman. 2017. Convolutional Neural Networks on Surfaces via Seamless Toric Covers. ACM Trans. Graph. 36, 4 (2017).

Lee Mosher. 1988. Tiling the Projective Foliation Space of a Punctured Surface. Trans. Amer. Math. Soc. (1988).

Patrick Mullen, Yiying Tong, Pierre Alliez, and Mathieu Desbrun. 2008. Spectral Conformal Parameterization. In Computer Graphics Forum, Vol. 27.

Todd Munson, Jason Sarich, Stefan Wild, Steve Benson, and Lois Curfman McInnes. 2014. Toolkit for Advanced Optimization (TAO) Users Manual. Technical Report ANL/MCS-TM-322 - Revision 3.5. Argonne National Laboratory.

Ashish Myles, Nico Pietroni, and Denis Zorin. 2014. Robust Field-Aligned Global Parametrization. ACM Transactions on Graphics 33, 4 (2014).

Robert C. Penner. 2012. Decorated Teichmüller Theory. European Mathematical Society (EMS), Zürich.

Roman Prosanov. 2020. Ideal Polyhedral Surfaces in Fuchsian Manifolds. Geometriae Dedicata 206 (2020)

Igor Rivin. 1994. Intrinsic Geometry of Convex Ideal Polyhedra in Hyperbolic 3-Space In Analysis, Algebra, and Computers in Mathematical Research (Luleå, 1992). Lect. Notes Pure Appl. Math., Vol. 156.

Igor Rivin. 1996. A Characterization of Ideal Polyhedra in Hyperbolic 3-Space. Annals of Mathematics. Second Series 143, 1 (1996).

M. Roček and R. M. Williams. 1984. The Quantization of Regge Calculus. Zeitschrift für Physik C Particles and Fields 21, 4 (1984).

Rohan Sawhney and Keenan Crane. 2017. Boundary First Flattening. ACM Transactions on Graphics 37, 1 (2017).

Marcus Schaefer, Eric Sedgwick, and Daniel Štefankovič. 2002. Algorithms for Normal Curves and Surfaces. In International Computing and Combinatorics Conference.

Nick Sharp and Keenan Crane. 2018. Variational Surface Cutting. ACM Trans. Graph 37, 4 (2018).

Nicholas Sharp and Keenan Crane. 2020. A Laplacian for Nonmanifold Triangle Meshes. In Computer Graphics Forum, Vol. 39.

Nicholas Sharp, Keenan Crane, et al. 2019a. Geometry-Central. (2019).

Nicholas Sharp, Yousuf Soliman, and Keenan Crane. 2019b. Navigating Intrinsic Triangulations. ACM Trans. Graph. 38, 4 (2019).

Nicholas Sharp, Yousuf Soliman, and Keenan Crane. 2019c. The Vector Heat Method. ACM Trans. Graph. 38, 3 (2019).

Alla Sheffer, Bruno Lévy, Maxim Mogilnitsky, and Alexander Bogomyakov. 2005. ABF++ Fast and Robust Angle Based Flattening. ACM Transactions on Graphics 24, 2 (2005).

Hanxiao Shen, Zhongshi Jiang, Denis Zorin, and Daniele Panozzo. 2019. Progressive Embedding. ACM Transactions on Graphics 38, 4 (2019).

Yousuf Soliman, Dejan Slepčev, and Keenan Crane. 2018. Optimal Cone Singularities for Conformal Flattening. ACM Trans. Graph. 37, 4 (2018).

Boris Springborn. 2019. Ideal Hyperbolic Polyhedra and Discrete Uniformization. Discrete \& Computational Geometry (2019).

Boris Springborn, Peter Schröder, and Ulrich Pinkall. 2008. Conformal Equivalence of Triangle Meshes. In ACM SIGGRAPH 2008 Papers.

Jian Sun, Tianqi Wu, Xianfeng David Gu, and Feng Luo. 2015. Discrete Conformal Deformation: Algorithm and Experiments. SIAM fournal on Imaging Sciences 8, 3 (2015)

Dylan Thurston and Qiaochu Yuan. 2012. Notes on Curves on Surfaces. (2012)

Marc Troyanov. 1991. Prescribing Curvature on Compact Surfaces with Conical Singularities. Trans. Amer. Math. Soc. 324, 2 (1991).

Ana Maria Vintescu, Florent Dupont, and Guillaume Lavoué. 2017. Least Squares Affine Transitions for Global Parameterization. (2017).

Tianqi Wu. 2014. Finiteness of Switches in Discrete Yamabe Flow. Ph.D. Dissertation Tsinghua University

BT Thomas Yeo, Mert R Sabuncu, Tom Vercauteren, Nicholas Ayache, Bruce Fischl, and Polina Golland. 2009. Spherical Demons: Fast Diffeomorphic Landmark-Free Surface Registration. IEEE transactions on medical imaging 29, 3 (2009).

Xiaokang Yu, Na Lei, Yalin Wang, and Xianfeng David Gu. 2017. Intrinsic 3D Dynamic Surface Tracking Based on Dynamic Ricci Flow and Teichmuller Map. In IEEE Vis.

Min Zhang, Ren Guo, Wei Zeng, Feng Luo, Shing-Tung Yau, and Xianfeng David Gu. 2014. The Unified Discrete Surface Ricci Flow. Graphical Models 76, 5 (2014).

Jiaran Zhou, Changhe Tu, Denis Zorin, and Marcel Campen. 2020. Combinatorial Construction of Seamless Parameter Domains. In Computer Graphics Forum, Vol. 39.

\section{A EXTRACTING GEODESICS}

Here we give a detailed description of how to extract geodesics from normal coordinates. Note that in this appendix, and in the supplement, we use $\langle u, v\rangle:=u_{1} v_{1}+u_{2} v_{2}+u_{3} v_{3}$ to denote the Euclidean inner product for vectors $u, v \in \mathbb{R}^{3}$, and $\langle u, v\rangle_{2,1}:=$ $u_{1} v_{1}+u_{2} v_{2}-u_{3} v_{3}$ for the Lorentz inner product.

\section{A.1 Projective Segment Intersection}

For several of our calculations, it will be useful to express the intersection between two planar line segments $a b$ and $c d$ in terms of the homogeneous coordinates of their endpoints. In particular, if $a, b, c, d \in \mathbb{R}^{3}$ are any homogeneous coordinates for the endpoints, we seek a solution to

$$
(1-t) a+t b=e^{u}((1-s) c+s d) .
$$

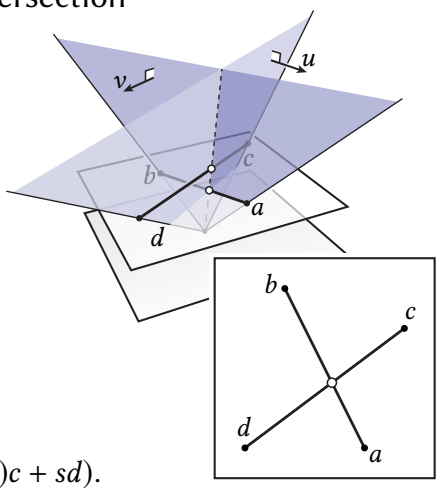

Letting $v:=a \times b$ and $w:=c \times d$, we can write the solution explicitly as

$$
t=\frac{\langle w, a\rangle}{\langle w, a-b\rangle}, \quad s=\frac{\langle v, c\rangle}{\langle v, c-d\rangle}, \quad u=\log \left(\frac{\langle v, d-c\rangle}{\langle w, a-b\rangle}\right) .
$$

\section{A.2 Tracing}

We here detail the algorithms for topological tracing, described in Section 6.1.1. In particular, Algorithm 2 considers the four cases depicted in Section 6.1.1. In case 1, several curves end at vertex $l$. Here there are three possibilities: the curve either continues through $i l$, terminates at $l$, or continues through $l j$. In case 2 , several curves end at $j$. This time, the curve must continue through $i j$. Similarly, in case 3 , several curves end at $i$, and the curve must continue through $\overrightarrow{l j}$. In case 4 , no curve ends at any vertex of $i l j$, and the curve continues through either $\overrightarrow{i l}$ or $\overrightarrow{l j}$.

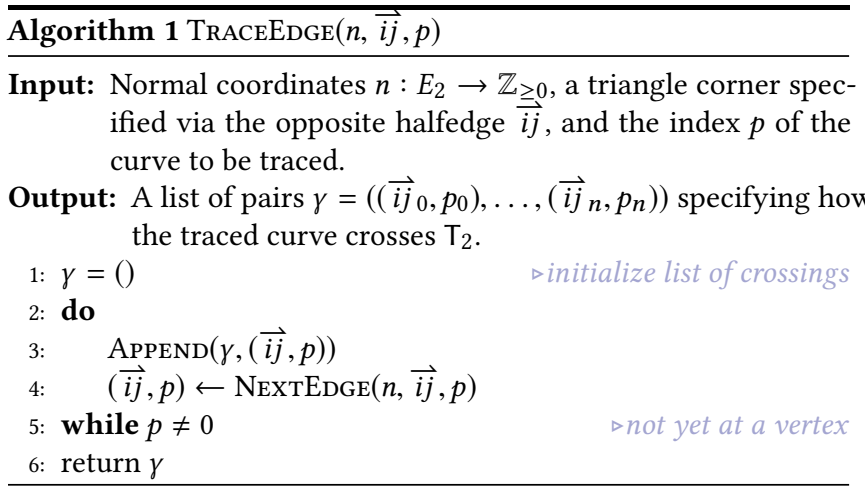




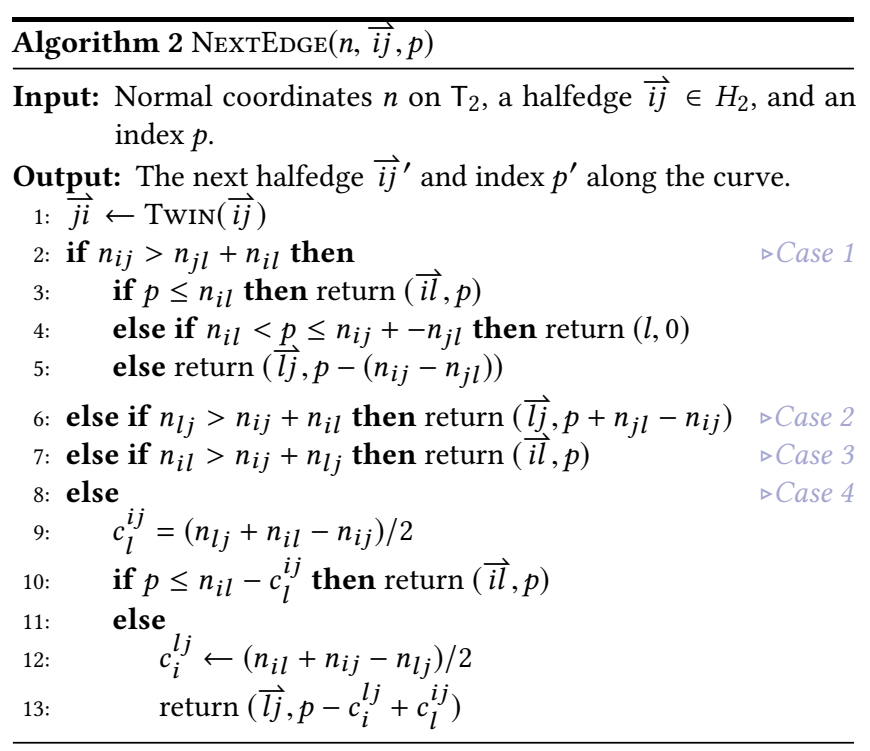

\section{A.3 Hyperbolic Geodesics}

We here describe how to extract the points where a hyperbolic geodesic $\gamma_{a b}$ intersects the sequence of halfedges computed via Algorithm 1. For each such halfedge $\overrightarrow{i j}$ we extract the barycentric coordinates $s, t$ along $\gamma_{a b}$ and $\overrightarrow{i j}$, resp., plus a log scale factor $u$ associated with the intersection. As in Section 6.2 we lay out a triangle strip, but this time place vertices on the light cone $\mathcal{L}$ (Section 3.3.1). As derived in the supplement, the first triangle $a i j$ has vertices

$$
\begin{aligned}
q_{a} & =\frac{2}{\sqrt{3}} \ell_{a i} \ell_{a j} \ell_{i j}^{-1}(1,0,1), \\
q_{i} & =\frac{2}{\sqrt{3}} \ell_{a i} \ell_{i j} \ell_{a j}^{-1}(\cos (2 \pi / 3), \sin (2 \pi / 3), 1), \\
q_{j} & =\frac{2}{\sqrt{3}} \ell_{a j} \ell_{i j} \ell_{a i}^{-1}(\cos (4 \pi / 3), \sin (4 \pi / 3), 1) .
\end{aligned}
$$

For any triangle $k j l$ following a known triangle $i j k$, we use the Ptolemy relation to get $\ell_{i l}$, then solve for the unknown position

$$
q_{l}=\frac{\ell_{i l}}{\ell_{i k} \ell_{i j}}\left(-\frac{\ell_{j l} \ell_{k l}}{\ell_{i l}} q_{i}+\frac{\ell_{i k} \ell_{k l}}{\ell_{j k}} q_{j}+\frac{\ell_{j l} \ell_{i j}}{\ell_{j k}} q_{k}\right) .
$$

This process repeats until we have laid out the whole strip, including the endpoints $q_{a}$ and $q_{b}$-to account for uniformization, we scale just these endpoints by $e^{-u_{a}}$ and $e^{-u_{b}}$, resp. If we then imagine that points $q \in \mathbb{R}^{3}$ in the light cone are homogeneous coordinates for points $x \in \mathbb{R}^{2}$ from the Beltrami-Klein model, then Equation 19 gives us the desired values $s, t$, and $u$ at each intersection.

\section{B REFINING FACES}

We here describe how to build a graph $\mathcal{G}=(\mathcal{V}, \mathcal{E})$ describing the connectivity of $S$ within a face $i j k \in \mathrm{T}^{B}$. Recall the nomenclature defined in Section 6.3. In Step I below we determine which edge points connect to form segments; in Step II we determine which segments intersect; in Step III we extract faces of $\mathcal{G}$. We first sort all edge points $p$ in counter-clockwise order (starting at any edge point), assigning them indices $\varphi_{p} \in \mathbb{Z}$ which provide a sort of combinatorial analogue to the angle around the boundary.

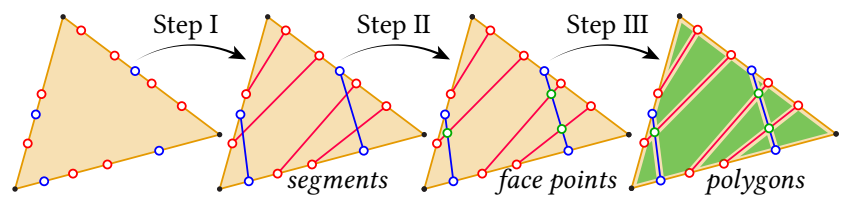

Fig. 34. The three stages of connectivity extraction.

Step I (Segments). We compute the segments from $\mathrm{T}^{A}$ and $\mathrm{T}^{C}$ independently, à la Sharp et al. [2019b, Section 3.4]. In each case, the number of edge points determine three normal coordinates $m_{i j} ; e_{i}^{j k}$ and $c_{i}^{j k}$ are then defined as in Section 5.1. If $e_{i}^{j k} \neq 0$, we connect the first $c_{j}^{k i}$ edge points along $j k$ to the first $c_{j}^{k i}$ edge points along $j i$, the next $e_{i}^{j k}$ edge points to vertex $i$, and the remaining ones to the edge points along $k i$ (see inset).

Step II (Segment-Segment Intersections). To find face points, we consider all pairs of segments $a b$ and $c d$ from $\mathrm{T}^{A}$ and $\mathrm{T}^{C}$, resp. Two segments cross if the values of $\varphi$ are interleaved, i.e., if they come in a (cyclic) order like $\varphi_{a}, \varphi_{c}, \varphi_{b}, \varphi_{d}$. If so, we add a new face point, maintaining a list of all face points along each segment, sorted by $\phi$ (which defines the fragments). After computing intersections, we use the $\phi$ values at segment endpoints to sort the fragments around each face point.

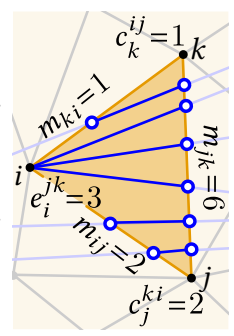

Step III (Polygons). To extract the final polygons, we iterate over fragments. For each fragment we visit the next vertex, then the next fragment in counter-clockwise order, until we return to the original fragment. If desired, the final (global) tessellation S can be collected in an ordinary mesh whose vertices consist of all face points, edge points, and vertices from the original vertex set $\mathrm{V}$.

Note that the sorting procedure in Step II is the only moment where floating point errors have any chance of invalidating the connectivity of the refinement. 